\title{
LINEAR COMPLEXITY SOLUTION OF \\ PARABOLIC INTEGRO-DIFFERENTIAL EQUATIONS
}

\author{
By
}

\section{Ana-Maria Matache \\ Christoph Schwab}

and

Thomas P. Wihler

IMA Preprint Series \# 1990

( September 2004)

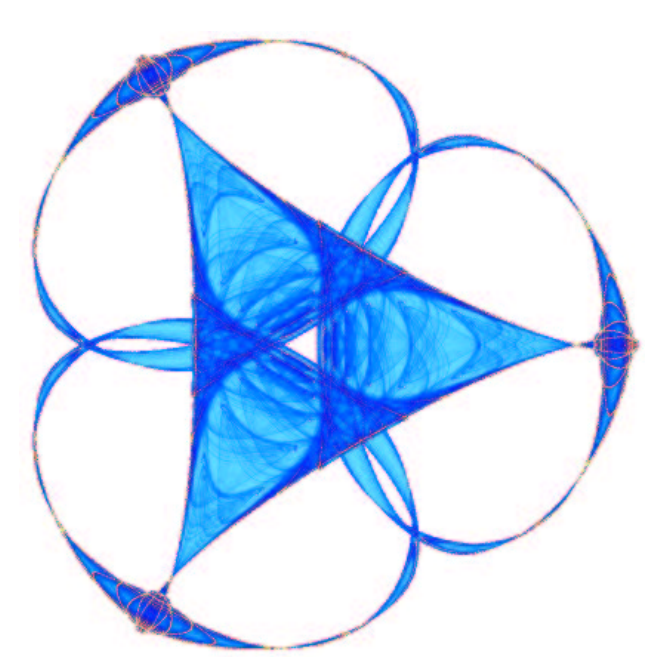

INSTITUTE FOR MATHEMATICS AND ITS APPLICATIONS

UNIVERSITY OF MINNESOTA

514 Vincent Hall

206 Church Street S.E.

Minneapolis, Minnesota 55455-0436

Phone: 612/624-6066 Fax: 612/626-7370

URL: http://www.ima.umn.edu 


\title{
LINEAR COMPLEXITY SOLUTION OF PARABOLIC INTEGRO-DIFFERENTIAL EQUATIONS
}

\author{
ANA-MARIA MATACHE, CHRISTOPH SCHWAB, AND THOMAS P. WIHLER
}

\begin{abstract}
The numerical solution of parabolic problems $u_{t}+\mathcal{A} u=0$ with a pseudodifferential operator $\mathcal{A}$ by wavelet discretization in space and $h p$ discontinuous Galerkin time stepping is analyzed. It is proved that an approximation for $u(T)$ can be obtained in $N$ points with accuracy $\mathcal{O}\left(N^{-p-1}\right)$ for any integer $p \geq 1$ in work and memory which grows logarithmically-linear in $N$.
\end{abstract}

\section{INTRODUCTION}

Since the fundamental work of E.B. Dynkin [16], R. Feynman [20] and M. Kac [25], it is well known that the solution of diffusion problems in a bounded domain $\Omega$ can be characterized as expectation of the exit of a Wiener process from $\Omega$ (see [21] for further details and references). This connection allows, on the one hand, to calculate expectations as solutions of deterministic partial differential equations, and, on the other hand, to characterize the solution of deterministic partial differential equations probabilistically. Both viewpoints have been used to obtain numerical solution methods.

This 'Feynman-Kac' connection was extended to more general Markov Processes where the sample paths are possibly discontinuous. Here, exit problems from a bounded domain $\Omega$ are related to the solution of a parabolic integro-differential equation,

$$
\begin{aligned}
\dot{u}(t)+\mathcal{A} u(t) & =0 & & \text { in } Q=J \times \Omega \\
u(0) & =u_{0} & & \text { in } \Omega,
\end{aligned}
$$

in the time interval $J=(0, T), 0<T<\infty$, with initial condition $u_{0} \in L^{2}(\Omega)$ and an integro-differential operator $\mathcal{A}$ arising as infinitesimal generator of the semigroup generated by a Markov Process (see [23, 24] for details and an extensive list of references). In particular, $\mathcal{A}$ is in general nonselfadjoint due to a drift term and due to asymmetry in the process' jump measure.

With the adoption of jump diffusion processes as price processes in mathematical finance, the corresponding option prices are characterized as solutions of parabolic integrodifferential equations (1.1)-(1.2). Accordingly, efficient numerical solutions of such equations is of interest in these applications.

A Monte Carlo simulation for the approximation of the solution of (1.1)-(1.2) gives the value of the solution in one point with accuracy $\mathcal{O}\left(N^{-1 / 2}\right)$ for a work of $N$ operations.

2000 Mathematics Subject Classification. 35K15, 45K05, 47G20, 65M12, 65M60, 65N30, 65T60.

Key words and phrases. Parabolic equations, integro-differential operators, discontinuous Galerkin methods, wavelets, matrix compression, GMRES, computational fi nance, option pricing.

Supported in part IHP Network Breaking Complexity of the EC (contract number HPRN-CT-2002-00286) with support by the Swiss Federal Offi ce for Science and Education under grant No. BBW 02.0418.

The third author was funded by the Swiss National Science Foundation (Grant PBEZ2-102321). 
In the special case where the process is a Lévy process, $\mathcal{A}$ is translation invariant and, accordingly, the solution of the parabolic problem (1.1)-(1.2) in $N$ points can be obtained by Fourier-Laplace transform techniques, see e.g. [9], in $\mathcal{O}(N)$ work where $N$ denotes the number of degrees of freedom used in $\Omega$. The accuracy of this approach is spectral.

For more general Feller processes, however, the infinitesimal generator $\mathcal{A}$ of the corresponding semigroup is an integro-differential operator which is not translation invariant and may not be time-homogeneous. The efficient numerical solution of (1.1)-(1.2) for such operators is the purpose of the present paper. We present an algorithm and prove that it allows to obtain approximate solution values in the interval $(0, T)$ for $T>0$ in $N$ points in $\Omega$ with accuracy $\mathcal{O}\left(N^{-p-1}\right)$ in work and memory of $\mathcal{O}\left(N(\log N)^{a}\right)$ operations with a small, positive integer $a$, for any integer order $p \geq 1$. As in $[33,42]$, we employ a wavelet discretization of $\mathcal{A}$ in $\Omega$ to compress the fully populated moment matrix of $\mathcal{A}$ and an exponentially convergent $h p$ discontinuous Galerkin time-stepping scheme to exploit the time analyticity of the semigroup generated by $\mathcal{A}$.

The outline of the paper is as follows. In Section 2, we give a variational formulation for the parabolic integro-differential equations studied in this paper and establish an appropriate functional setting for our analysis. Section 3 describes the space and time discretization, in particular the wavelet compression of the moment matrix of $\mathcal{A}$ and the high order discontinuous Galerkin time stepping scheme, and establishes error estimates. Section 4 presents the actual solution algorithm and addresses the log-linear complexity. Section 5 is devoted to the proofs of the compression error estimates, while Section 6 gives some applications to mathematical finance. Finally, we summarize our work in Section 7.

\section{Parabolic Evolution Problems}

Let $\Omega=(a, b),-\infty<a<b<\infty$, be a bounded interval in $\mathbb{R}$. By $L^{2}(\Omega)$, we denote the usual space of square integrable functions on $\Omega$ with the inner product $(\cdot, \cdot)$ and the norm $\|\cdot\|$, and by $H^{s}(\Omega), s \geq 0$, the corresponding Sobolev spaces equipped with the standard norms $\|\cdot\|_{H^{s}(\Omega)}$ (see, e.g. [1]). In addition, we define the (complex) Hilbert spaces

$$
\widetilde{H}^{s}(\Omega)=\left\{\left.u\right|_{\Omega}: u \in H^{s}(\mathbb{R}),\left.u\right|_{\mathbb{R} \backslash \Omega}=0\right\}, \quad 0 \leq s \leq 1 .
$$

Note that, if $s \neq \frac{1}{2}$, then $\widetilde{H}^{s}(\Omega)$ coincides with the closure of $\mathcal{C}_{0}^{\infty}(\Omega)$ with respect to the norm $\|\cdot\|_{H^{s}(\Omega)}$. In the case $s=\frac{1}{2}$, we have the identity $\widetilde{H}^{\frac{1}{2}}(\Omega)=H_{00}^{\frac{1}{2}}(\Omega)$; see [30] for details.

We identify $L^{2}(\Omega)$ with its dual and define, for $0<\rho \leq 2$,

$$
V=\widetilde{H}^{\frac{\rho}{2}}(\Omega) .
$$

There hold the dense injections (Gelfand triple) $V \stackrel{d}{\hookrightarrow} L^{2}(\Omega) \stackrel{d}{\hookrightarrow} V^{*}$, where $V^{*}=$ $H^{-\frac{\rho}{2}}(\Omega)$ denotes the dual space of $V$. Furthermore, let $\langle\cdot, \cdot\rangle_{V^{*} \times V}$ be the duality pairing in $V^{*} \times V$, and $\|\cdot\|_{V},\|\cdot\|_{V^{*}}$ the norms in $V$ and $V^{*}$, respectively.

We consider a linear, possibly nonlocal operator $\mathcal{A} \in \mathcal{L}\left(V, V^{*}\right)$ of order $\rho$,

$$
\langle\mathcal{A} u, v\rangle_{V^{*} \times V}=a(u, v), \quad u, v \in V .
$$

with associated sesquilinear form $a: V \times V \rightarrow \mathbb{C}$ which we assume to be continuous, i.e. there exists a constant $\alpha>0$ such that

$$
|a(u, v)| \leq \alpha\|u\|_{V}\|v\|_{V}, \quad \forall u, v \in V .
$$


Moreover, we assume that, for some constants $\beta>0, \gamma \geq 0$, the form $a(\cdot, \cdot)$ satisfies a Gårding inequality, i.e.,

$$
\operatorname{Re} a(u, u) \geq \beta\|u\|_{V}^{2}-\gamma\|u\|^{2}, \quad \forall u \in V .
$$

We may and will assume $\gamma=0$ in (2.2), i.e., $a$ is coercive on $V$ :

$$
\operatorname{Re} a(u, u) \geq \beta\|u\|_{V}^{2}, \quad \forall u \in V .
$$

Indeed, the transform $\tilde{u}=e^{-\gamma t} u$ results in the equation

$$
\begin{aligned}
\tilde{u}^{\prime}(t)+(\mathcal{A}+\gamma \mathbb{I}) \tilde{u}(t) & =0 & & \text { in } Q=J \times \Omega \\
\tilde{u}(0) & =u_{0} & & \text { in } \Omega,
\end{aligned}
$$

and by (2.2) the operator $\mathcal{A}+\gamma \mathbb{I}$ is coercive and satisfies (2.3) with coercivity constant $\beta$.

The operator $\mathcal{A}$ needs not to be self-adjoint, i.e. $a(\cdot, \cdot)$ can be non-symmetric.

To describe the smoothness of the initial data $u_{0}$ in (1.2), we introduce spaces between $L^{2}(\Omega)$ and $V$, defined by the $K$-method of interpolation [30, 40], as

$$
\mathcal{V}_{\theta}=\left[L^{2}(\Omega), V\right]_{\theta, 2}, \quad 0 \leq \theta \leq 1,
$$

with the usual convention that $\mathcal{V}_{0}=L^{2}(\Omega)$ and $\mathcal{V}_{1}=V$.

Under the above conditions, problem (1.1)-(1.2) has a unique (weak) solution $u(t)$, and there holds the a priori estimate (see [30])

$$
\|u\|_{\mathcal{C}\left(\bar{J}, L^{2}(\Omega)\right)}+\|u\|_{L^{2}(J, V)}+\|\dot{u}\|_{L^{2}\left(J, V^{*}\right)} \leq C\left\|u_{0}\right\|_{L^{2}(\Omega)} .
$$

Specifically, the operator $\mathcal{A}$ is assumed to be the sum of a second order differential operator and of a classical pseudo-differential operator with a (real) distributional kernel $k(x, x-y) \in \mathcal{D}^{\prime}(\Omega \times \Omega)$, with associated sesquilinear form given by

$$
a(u, v)=\langle\mathcal{A} u, v\rangle_{V^{*} \times V}=\int_{\Omega} \int_{\Omega} k(x, x-y) u(x) \overline{v(y)} d x d y .
$$

The kernel $k(x, z) \in \mathcal{C}^{\infty}(\Omega \times \mathbb{R} \backslash\{z=0\} ; \mathbb{R})$ is assumed to satisfy Caldéron-Zygmund type estimates, i.e. $\forall \alpha, \beta \in \mathbb{N}_{0}$, there holds

$$
\left|\partial_{x}^{\alpha} \partial_{z}^{\beta} k(x, z)\right| \leq C(\alpha, \beta)|z|^{-1-\rho-\alpha-\beta}, \quad z \neq 0,
$$

uniformly with respect to $x \in \Omega$.

\section{DisCRETIZATION}

We discretize the parabolic equation (1.1)-(1.2) in space and time using a wavelet finite element method on $\Omega=(a, b)$, and a discontinuous Galerkin (dG) scheme on $J=(0, T)$.

In our analysis, we will need to consider functions in $V$ with additional regularity. Therefore, for $s \geq 0$, we define the spaces

$$
\mathcal{H}^{s}(\Omega)= \begin{cases}\widetilde{H}^{s}(\Omega) & \text { for } s \leq \frac{\rho}{2}, \\ V \cap H^{s}(\Omega) & \text { for } s>\frac{\rho}{2} .\end{cases}
$$

By $\|\cdot\|_{s}$, we denote the corresponding norm in $\mathcal{H}^{s}(\Omega)$. 
3.1. Spatial Semi-Discretization. We first discretize the parabolic problem (1.1)-(1.2) with respect to the space variable. Hence, let $\mathcal{T}^{0}$ be a fixed coarse partition of $\Omega$. Furthermore, define the mesh $\mathcal{T}^{l}$, for $l>0$, recursively by bisection of each interval in $\mathcal{T}^{l-1}$. We assume that our computational mesh $\mathcal{T}_{h}$ (with mesh size $h$ ) is obtained in this way as $\mathcal{T}^{L}$, for some $L>0$, with $h=C 2^{-L}$.

The finite element space $V_{h} \subset V$ used for the spatial discretization is the space of all continuous piecewise polynomials of degree $p \geq 1$ on the triangulation $\mathcal{T}_{h}$ which vanish on the boundary $\partial \Omega$. In the same way, we define the spaces $V^{l}$ corresponding to the triangulation $\mathcal{T}^{l}$, so that we have $V^{0} \subset V^{1} \subset \cdots \subset V^{L}=V_{h}$. Let $N^{l}=\operatorname{dim} V^{l}$ and $N=\operatorname{dim} V_{h}=N^{L}=C 2^{L}$.

The semi-discrete problem corresponding to (1.1)-(1.2) reads: Given $u_{0} \in L^{2}(\Omega)$, find $u_{h} \in H^{1}\left(J, V_{h}\right)$ such that

$$
\frac{d}{d t}\left(u_{h}, v_{h}\right)+a\left(u_{h}, v_{h}\right)=0, \quad \forall v_{h} \in V_{h},
$$

and

$$
u_{h}(0)=P_{h} u_{0} .
$$

Here, $P_{h}$ is the $L^{2}$ projection onto $V_{h}$.

We have the following a priori result on the spatial semi-discretization:

Theorem 3.1. Let (2.1) and (2.3) be satisfied. Furthermore, we assume that the operator $\mathcal{A}$ is of the form $\mathcal{A}=\mathcal{A}_{0}+\mathcal{B}$, where $\mathcal{A}_{0}=C_{0}(-\Delta)^{\frac{\rho}{2}}, C_{0}>0$, is a Riesz potential, and $\mathcal{B}: V \rightarrow V^{*}$ is a compact perturbation. Then, for $t>0$, there holds

$$
\left\|u(t)-u_{h}(t)\right\| \leq C \min \left\{1, h^{p+1} t^{-\frac{p+1}{\rho}}\right\} .
$$

Here, $C>0$ is a constant independent of $h$ and $t$, and $u$, $u_{h}$ are the solutions of (1.1)-(1.2) and (3.1)-(3.2), respectively.

The proof is postponed to Section 5.1.

We remark that the assumption on the principal part $\mathcal{A}_{0}$ in the preceding theorem is satisfied for most operators occurring in practice.

3.1.1. Wavelet basis. In order to compute a fully discrete approximation (in space and time) to the parabolic problem (1.1)-(1.2), systems of linear equations have to be solved in each time step (of the corresponding time discretization). Due to the nonlocal character of the operator $\mathcal{A}$ the matrices corresponding to these linear systems are typically fully populated. Therefore, matrix compression techniques, transforming the dense matrices into sparse ones, have to be employed. The compressed systems may then be manipulated in linear complexity.

Using an appropriate basis for the finite dimensional space $V_{h}$, we will be able to represent the sesquilinear form $a(\cdot, \cdot)$ as a matrix whose entries are mostly negligible, thereby allowing for matrix compression techniques.

More precisely, we consider so-called biorthogonal wavelets

$$
\left\{\psi_{j}^{l}\right\}_{j, l}, \quad l=0,1, \ldots, \quad j=1,2, \ldots, M^{l},
$$

which allow for optimal preconditioning. They satisfy the following properties (see e.g. [10]):

(1) $V_{h}=V^{L}=\operatorname{span}\left\{\psi_{j}^{l} \mid 0 \leq l \leq L, 1 \leq j \leq M^{l}\right\}$.

(2) The function $\psi_{j}^{l}$ has support $S_{j}^{l}=\operatorname{supp} \psi_{j}^{l}$ of diameter bounded by $C 2^{-l}$. 
(3) Wavelets $\psi_{j}^{l}$ with $\overline{S_{j}^{l}} \cap \partial \Omega=\emptyset$ have vanishing moments up to order $p$, i.e., $\left(\psi_{j}^{l}, q\right)=0$ for all polynomials $q$ of degree $p$ or less.

(4) The functions $\psi_{j}^{l}$ for $l \geq l_{0}$ are obtained by scaling and translation of the functions $\psi_{j}^{l_{0}}$.

(5) For all $v_{h}=\sum_{l=0}^{L} \sum_{j=1}^{M^{l}} v_{j}^{l} \psi_{j}^{l} \in V_{h}$, there holds the norm equivalence

$$
\left\|v_{h}\right\|_{s}^{2} \cong \sum_{l=0}^{L} \sum_{j=1}^{M^{l}}\left|v_{j}^{l}\right|^{2} 2^{2 l s},
$$

for all $0 \leq s \leq 1$.

Remark 3.2. The biorthogonal wavelets in the case $p=1$ are continuous, piecewise linear spline wavelets vanishing outside $\Omega=(0,1)$ (for more general domains $\Omega=(a, b)$, they are obtained by simple scalings). The interior wavelets have two vanishing moments and are obtained from the mother wavelet $\psi(x)$ which takes the values $\left(0,-\frac{1}{2}, 1,-\frac{1}{2}, 0\right)$ at $\left(0, \frac{1}{4}, \frac{1}{2}, \frac{3}{4}, 1\right)$ by scaling and translations: $\psi_{j}^{l}(x):=\psi\left(2^{l-1} x-(2 j-1) 2^{-2}\right)$ for $1 \leq j \leq 2^{l}-2$ and $l \geq 2$. The boundary wavelets are constructed from the continuous, piecewise linear functions $\psi_{*}$, with values $\left(0,1,-\frac{1}{2}, 0\right)$ at $\left(0, \frac{1}{4}, \frac{1}{2}, \frac{3}{4}\right)$, and $\psi^{*}$, taking values $\left(0,-\frac{1}{2}, 1,0\right)$ at $\left(\frac{1}{4}, \frac{1}{2}, \frac{3}{4}, 1\right): \psi_{0}^{l}=\psi_{*}\left(2^{l-1} x\right)$ and $\psi_{2^{l}-1}^{l}=\psi^{*}\left(2^{l-1} x-2^{l-1}+1\right)$. Higher order spline wavelets with analogous properties have been given in [14].

We note that a function $v \in V$ can be represented in the form

$$
v=\sum_{l=0}^{\infty} \sum_{j=1}^{M^{l}} v_{j}^{l} \psi_{j}^{l} .
$$

This makes it possible to define an approximation operator $Q_{h}: \mathcal{H}^{s}(\Omega) \rightarrow V_{h}$, defined by

$$
Q_{h} v=\sum_{l=0}^{L} \sum_{j=1}^{M^{l}} v_{j}^{l} \psi_{j}^{l} .
$$

For $0 \leq s \leq \frac{\rho}{2} \leq t \leq p+1$, we have have the approximation property

$$
\left\|v-Q_{h} v\right\|_{s} \leq C h^{t-s}\|v\|_{t} .
$$

3.1.2. Matrix Compression. The sesquilinear form $a$ on $V_{h} \times V_{h}$ corresponds to a matrix A with entries $A_{(l, j),\left(l^{\prime}, j^{\prime}\right)}=a\left(\psi_{j^{\prime}}^{l^{\prime}}, \psi_{j}^{l}\right)$ which, due to the Caldéron-Zygmund estimates (2.6), decay with increasing distance of their supports. Hence, we can define a compressed matrix $\widetilde{\mathbf{A}}$ and a corresponding sesquilinear form $\widetilde{a}$ by replacing some of the small entries in A with zero:

$$
\widetilde{A}_{(j, l),\left(j^{\prime}, l^{\prime}\right)}= \begin{cases}A_{(j, l),\left(j^{\prime}, l^{\prime}\right)} & \text { if } \operatorname{dist}\left(S_{j}^{l}, S_{j^{\prime}}^{l^{\prime}}\right) \leq \delta_{l, l^{\prime}} \text { or } S_{j}^{l} \cap \partial \Omega \neq \emptyset, \\ 0 & \text { otherwise. }\end{cases}
$$

Here, the truncation parameters $\delta_{l, l^{\prime}}$ are given by

$$
\delta_{l, l^{\prime}}:=c \max \left\{2^{-L+\widehat{\alpha}\left(2 L-l-l^{\prime}\right)}, 2^{-l}, 2^{-l^{\prime}}\right\},
$$

with some parameters $c>0$ and $0<\widehat{\alpha} \leq 1$, and $S_{j}^{l}=\operatorname{supp} \psi_{j}^{l}$.

By continuity and coercivity of the sesquilinear form $a$, we have the norm equivalence

$$
\|u\|_{a}=|a(u, u)|^{\frac{1}{2}} \cong\|u\|_{V} .
$$

As in [42, Proposition 3.2], we have 
Proposition 3.3. Assume $c$ in (3.9) is sufficiently large. Then, there exists $0<\widetilde{\beta} \leq \widetilde{\alpha}<\infty$ independent of $h$ such that

$$
\left|\widetilde{a}\left(u_{h}, v_{h}\right)\right| \leq \widetilde{\alpha}\left\|u_{h}\right\|_{a}\left\|v_{h}\right\|_{a}
$$

and

$$
\operatorname{Re} \widetilde{a}\left(u_{h}, u_{h}\right) \geq \widetilde{\beta}\left\|u_{h}\right\|_{a}^{2},
$$

for all $u_{h}, v_{h} \in V_{h}$.

Moreover, there holds [41, 42]:

Proposition 3.4. Assume $c$ in (3.9) is sufficiently large. Then, there exists $0<\delta<1$ independent of $h$ such that for all $L>0$ the following consistency condition is satisfied:

$$
\left|a\left(u_{h}, v_{h}\right)-\widetilde{a}\left(u_{h}, v_{h}\right)\right| \leq \delta\left\|u_{h}\right\|_{a}\left\|v_{h}\right\|_{a}, \quad \forall u_{h}, v_{h} \in V_{h} .
$$

In addition, if

$$
\widehat{\alpha}>\frac{2 p+2}{2 p+2+\rho}
$$

where $\widehat{\alpha}$ is the constant from (3.9), then, there holds

$$
\left|a\left(Q_{h} u, v_{h}\right)-\widetilde{a}\left(Q_{h} u, v_{h}\right)\right| \leq C h^{s-\frac{\rho}{2}}\|u\|_{s}\left\|v_{h}\right\|_{V}, \quad \forall u \in \mathcal{H}^{s}(\Omega), \forall v_{h} \in V_{h},
$$

and

$\left|a\left(Q_{h} u, Q_{h} v\right)-\widetilde{a}\left(Q_{h} u, Q_{h} v\right)\right| \leq C h^{s+s^{\prime}-\rho}\|u\|_{s}\|v\|_{s^{\prime}}, \quad \forall u \in \mathcal{H}^{s}(\Omega), \forall v \in \mathcal{H}^{s^{\prime}}(\Omega)$,

for all $\frac{\rho}{2} \leq s, s^{\prime} \leq p+1$. Here, $Q_{h}$ is the projection operator from (3.6).

The matrix compression (3.8) reduces the number of nonzero elements from $N^{2}$ in $\mathbf{A}$ to $N$ times a logarithmic term in $\widetilde{\mathbf{A}}$; see [37]. More precisely, there holds:

Proposition 3.5. For $\widehat{\alpha}<1$, the number of nonzero elements in $\widetilde{\mathbf{A}}$ is $\mathcal{O}(N \log N)$. If $\widehat{\alpha}=1$, then the number of nonzero elements in $\widetilde{\mathbf{A}}$ is $\mathcal{O}\left(N(\log N)^{2}\right)$.

3.1.3. Perturbed Spatial Semi-Discretization. The matrix compression from the previous section induces, instead of (3.1)-(3.2), a perturbed spatial semi-discretization of (1.1)(1.2): find $\widetilde{u}_{h} \in V_{h}$ such that

$$
\frac{d}{d t}\left(\widetilde{u}_{h}, v_{h}\right)+\widetilde{a}\left(\widetilde{u}_{h}, v_{h}\right)=0, \quad \forall v_{h} \in V_{h} .
$$

and

$$
\widetilde{u}_{h}(0)=P_{h} u_{0} .
$$

We have the following analog to Theorem 3.1:

Theorem 3.6. Let the assumptions of Theorem 3.1 be satisfied. Moreover, let (3.10)-(3.15) hold. Then, for $t>0$, we have the following a priori error estimate for the perturbed semidiscrete problem (3.16)-(3.17):

$$
\left\|u(t)-\widetilde{u}_{h}(t)\right\| \leq C \min \left\{1, h^{p+1} t^{-\frac{p+1}{\rho}}\right\},
$$

where $C>0$ is a constant independent of $h$ and $t$, and $u$ is the solution of (1.1)-(1.2).

The proof of this result is worked out in Section 5.2. 
3.2. Discontinuous Galerkin Time Discretization. In order to obtain a fully discrete approximation (in space and time) to the parabolic problem (1.1)-(1.2), we discretize (3.16)(3.17) in time using a discontinuous Galerkin method following [39].

For $0<T<\infty$ and $M \in \mathbb{N}$, let $\mathcal{M}=\left\{I_{m}\right\}_{m=1}^{M}$ be a partition of $J=(0, T)$ into $M$ subintervals $I_{m}=\left(t_{m-1}, t_{m}\right), m=1,2, \ldots, M$ with $0=t_{0}<t_{1}<t_{2}<\ldots<t_{M}=T$. Moreover, denote by $k_{m}=t_{m}-t_{m-1}$ the length of $I_{m}$.

For $u \in H^{1}\left(\mathcal{M}, V_{h}\right)=\left\{v \in L^{2}\left(J, V_{h}\right):\left.v\right|_{I_{m}} \in H^{1}\left(I_{m}, V_{h}\right), m=1,2, \ldots, M\right\}$, define the one-sided limits

$$
\begin{array}{ll}
u_{m}^{+}=\lim _{s \rightarrow 0^{+}} u\left(t_{m}+s\right), & m=0,1, \ldots, M-1, \\
u_{m}^{-}=\lim _{s \rightarrow 0^{+}} u\left(t_{m}-s\right), & m=1,2, \ldots, M,
\end{array}
$$

and the jumps

$$
\llbracket u \rrbracket_{m}=u_{m}^{+}-u_{m}^{-}, \quad m=1,2, \ldots, M-1 .
$$

In addition, to each time interval $I_{m}$, a polynomial degree (approximation order) $r_{m} \geq 0$ is associated. These numbers are stored in the degree vector $\underline{r}=\left\{r_{m}\right\}_{m=1}^{M}$. Then, the following space being used for the discontinuous Galerkin $(\mathrm{dG})$ method is introduced:

$$
\mathcal{S}^{\underline{r}}\left(\mathcal{M}, V_{h}\right)=\left\{u \in L^{2}\left(J, V_{h}\right):\left.u\right|_{I_{m}} \in \mathcal{P}_{r_{m}}\left(I_{m}, V_{h}\right), m=1,2, \ldots, M\right\},
$$

where $\mathcal{P}_{r_{m}}\left(I_{m}\right)$ denotes the space of polynomials of degree at most $r_{m}$ on $I_{m}$.

With these definitions, the fully discrete $\mathrm{dG}$ scheme for the solution of problem (1.1)(1.2), respectively (3.16)-(3.17) reads as follows: find $\widetilde{U}_{h}^{d G} \in \mathcal{S} \underline{\underline{r}}\left(\mathcal{M}, V_{h}\right)$ such that for all $W \in \mathcal{S} \underline{r}\left(\mathcal{M}, V_{h}\right)$, there holds

$$
\widetilde{B}_{d G}\left(\widetilde{U}_{h}^{d G}, W\right)=F_{d G}(W),
$$

where

$$
\widetilde{B}_{d G}(U, W)=\sum_{m=1}^{M} \int_{I_{m}}((\dot{U}, W)+\widetilde{a}(U, W)) d t+\sum_{m=1}^{M-1}\left(\llbracket U \rrbracket_{m}, W_{m}^{+}\right)+\left(U_{0}^{+}, W_{0}^{+}\right),
$$

and

$$
F_{d G}(W)=\left(P_{h} u_{0}, W_{0}^{+}\right) .
$$

We recall the following results from [38]:

\section{Proposition 3.7.}

(a) The solution $\widetilde{U}_{h}^{d G} \in \mathcal{S} \underline{\underline{r}}\left(\mathcal{M}, V_{h}\right)$ of the $d G$ method (3.19) is uniquely defined.

(b) The solution $\widetilde{u}_{h}$ of (3.16)-(3.17) satisfies the $d G$ formulation (3.19), i.e. we have the Galerkin orthogonality

$$
\widetilde{B}_{d G}\left(\widetilde{u}_{h}-\widetilde{U}_{h}^{d G}, W\right)=0,
$$

for all $W \in \mathcal{S} \underline{\underline{r}}\left(\mathcal{M}, V_{h}\right)$.

Remark 3.8. The dG method (3.19) can be interpreted as a time stepping scheme of variable step size $k_{m}$ and orders $r_{m}$. Indeed, assuming that $\widetilde{U}_{h}^{d G}$ is known on the time intervals $I_{m}=\left(t_{m-1}, t_{m}\right), m=1,2, \ldots, n-1$, we may find $\widetilde{U}_{h}^{d G} \in \mathcal{P}_{r_{n}}\left(I_{n}, V_{h}\right), 1 \leq n \leq M$, by solving the variational problem

$$
\int_{I_{n}}\left(\left(\partial_{t} \widetilde{U}_{h}^{d G}, W\right)+\widetilde{a}\left(\widetilde{U}_{h}^{d G}, W\right)\right) d t+\left(\widetilde{U}_{n-1}^{d G+}, W_{n-1}^{+}\right)=\left(\widetilde{U}_{n-1}^{d G-}, W_{n-1}^{+}\right),
$$


for all $W \in \mathcal{P}_{r_{n}}\left(I_{n}, V_{h}\right)$. Here, we set $\widetilde{U}_{0}^{d G-}=P_{h} u_{0}$.

For $v_{h} \in V_{h}$ and $U \in \mathcal{S} \underline{r}\left(\mathcal{M}, V_{h}\right)$, we introduce the norms

$$
\left\|v_{h}\right\|_{\widetilde{a}}=\left|\widetilde{a}\left(v_{h}, v_{h}\right)\right|^{\frac{1}{2}},
$$

and

$$
\|U\|_{d G}^{2}=\sum_{m=1}^{M} \int_{I_{m}}\|U\|_{\widetilde{a}}^{2} d t+\frac{1}{2}\left(\left\|U_{0}^{+}\right\|^{2}+\sum_{m=1}^{M-1}\left\|\llbracket U \rrbracket_{m}\right\|^{2}+\left\|U_{M}^{-}\right\|^{2}\right) .
$$

Then, we have the following stability result for the $\mathrm{dG}$ scheme. Its proof is similar as the proof of [32, Proposition 4.8].

Proposition 3.9. The solution $\widetilde{U}_{h}^{d G} \in \mathcal{S} \underline{r}\left(\mathcal{M}, V_{h}\right)$ of the $d G$ method (3.19) satisfies the stability estimate

$$
\left\|\widetilde{U}_{h}^{d G}\right\|_{d G} \leq C\left\|u_{0}\right\|
$$

with a constant $C>0$ independent of $h$ and of $T$.

3.3. Convergence of the Fully Discrete Scheme. The solution operator of the parabolic problem (1.1)-(1.2) generates a holomorphic semi-group (see e.g. [38]). Therefore, the solution $u(t)$ of (1.1)-(1.2) is analytic with respect to $t$ for all $t>0$. However, due to the non-smoothness of the initial data, the solution may be singular at $t=0$. The aim of this section is to show how, by the use of so-called geometric time partitions and linearly increasing polynomial degrees in the time discretization, the low regularity of the solution at $t=0$ can be resolved.

Definition 3.10. A partition $\mathcal{M}_{M, \gamma}=\left\{I_{m}\right\}_{m=1}^{M}$ in $J=(0, T), T>0$, is called geometric with $M$ time steps $I_{m}=\left(t_{m-1}, t_{m}\right), m=1,2, \ldots, M$, and grading factor $\gamma \in(0,1)$, if

$$
t_{0}=0, \quad t_{m}=T \gamma^{M-m}, \quad 1 \leq m \leq M .
$$

Definition 3.11. A polynomial degree vector $\underline{r}$ is called linear with slope $\mu>0$ on $\mathcal{M}_{M, \gamma}$, if $r_{1}=0$ and $r_{m}=\lfloor\mu m\rfloor, m=2, \ldots, M$, where $\lfloor\mu m\rfloor=\max \left\{q \in \mathbb{N}_{0}: q \leq \mu m\right\}$.

We have the following a priori error estimate on the fully discrete (perturbed) $d G$ method.

Theorem 3.12. Let $\rho \in(0,2]$, and $u$ be the solution of the parabolic problem (1.1)-(1.2) on $J \times \Omega=(0, T) \times(a, b)$, with initial data $u_{0} \in \mathcal{V}_{\theta}$, for some $0<\theta \leq 1$. Moreover, let the assumptions of Theorem 3.6 be fulfilled, and $0<\gamma<1$. Then, there exist $\mu_{0}, \kappa_{0}>0$ such that for all geometric partitions $\mathcal{M}_{M, \gamma}$ (cf. Definition 3.10) with $M \geq \kappa_{0}|\log h|$, and all polynomial degree vectors $\underline{r}$ on $\mathcal{M}_{M, \gamma}\left(c f\right.$. Definition 3.11) with slope $\mu>\mu_{0}$, the fully discrete solution $\widetilde{U}_{h}^{d G}$ to (1.1)-(1.2) obtained by (3.19) on the finite element space $\mathcal{S} \underline{-}\left(\mathcal{M}_{M, \gamma}, V_{h}\right)$ satisfies the a priori error estimate

$$
\left\|u(T)-\widetilde{U}_{h}^{d G}(T)\right\| \leq C T^{-\frac{p+1}{\rho}} h^{p+1} .
$$

Here, $h$ and $p$ are the mesh size and the polynomial degree of the spatial discretization, respectively, and $C>0$ is a constant independent of $h$.

Proof. Thanks to the triangle inequality, we have

$$
\left\|u(T)-\widetilde{U}_{h}^{d G}(T)\right\| \leq\left\|u(T)-\widetilde{u}_{h}(T)\right\|+\left\|\widetilde{u}_{h}(T)-\widetilde{U}_{h}^{d G}(T)\right\|=: T_{1}+T_{2},
$$

where $\widetilde{u}_{h}$ is the solution of the semi-discrete problem (3.16)-(3.17).

The term $T_{1}$ is estimated using Theorem 3.6. In order to obtain a bound for $T_{2}$, we note that, on geometrically refined meshes with linearly increasing polynomial degrees (in the 
dG time discretization), $T_{2}$ converges at an exponential rate with respect to the number of degrees of freedom $\widehat{N}=\operatorname{dim} \mathcal{S}-\underline{\underline{r}}\left(\mathcal{M}_{M, \gamma}, V_{h}\right) / \operatorname{dim} V_{h}$ in the dG time discretization. More precisely, there holds $T_{2} \leq C e^{-b \sqrt{\widehat{N}}}$; see $[32,38,43]$ for details. Hence, we have

$$
\left\|u(T)-\widetilde{U}_{h}^{d G}(T)\right\| \leq T_{1}+T_{2} \leq C\left(h^{p+1}+e^{-b \sqrt{\widehat{N}}}\right) .
$$

Noticing that $\sqrt{\widehat{N}} \geq C M \geq C \kappa_{0}|\log h|$, the proof follows if $\kappa_{0}$ is chosen sufficiently large.

\section{Solution Algorithm}

We will now study the linear systems resulting from the dG method (3.19). We show that they may be solved iteratively, without causing a loss in the rates of convergence in the error estimate (3.24), by the use of an incomplete GMRES method. Furthermore, we prove that the overall complexity of the fully discrete dG method is linear (up to some logarithmic terms). Since these matters have already been studied in [32, 43], we restrict ourselves to a brief presentation of the results there.

4.1. Derivation of the Linear Systems. The dG time stepping scheme (3.23) corresponds to a linear system of size $\left(r_{m}+1\right) N^{L}$ to be solved in each time step $m=1,2, \ldots, M$. Here and in what follows, in order to clarify the dependence on the refinement level $L$ explicitly, we denote by $N^{L}$ the dimension of the finite element space $V_{h}=V^{L}$.

For $1 \leq m \leq M$, let $\left\{\phi_{j}=\sqrt{j+\frac{1}{2}} L_{j}\right\}_{j=0}^{r_{m}}$, where $L_{j}$ is the $j$-th Legendre polynomial on $(-1,1)$ (normalized such that $L_{j}(1)=1$ ), be a basis of the polynomial space $\mathcal{P}_{r_{m}}(-1,1)$. Then, the temporal shape functions on the time interval $I_{m}$ are given by $\phi_{j} \circ F_{m}^{-1}$, where the mapping $F_{m}:(-1,1) \rightarrow I_{m}$ is given by

$$
t=F_{m}(\hat{t})=\frac{1}{2}\left(t_{m-1}+t_{m}\right)+\frac{1}{2} k_{m} \hat{t}, \quad k_{m}=t_{m}-t_{m-1}, \quad \hat{t} \in(-1,1) .
$$

Writing $\widetilde{U}_{h, m}^{d G}(x, t)=\left.\widetilde{U}_{h}^{d G}\right|_{I_{m}}(x, t)$ and $W_{m}=\left.W\right|_{I_{m}}$ in (3.23) as

$$
\begin{aligned}
\widetilde{U}_{h, m}^{d G}(x, t) & =\sum_{j=0}^{r_{m}} \widetilde{U}_{h, m, j}^{d G}(x)\left(\phi_{j} \circ F_{m}^{-1}\right)(t), \\
W_{m}(x, t) & =\sum_{j=0}^{r_{m}} W_{m, j}(x)\left(\phi_{j} \circ F_{m}^{-1}\right)(t),
\end{aligned}
$$

the variational formulation (3.23) reads: find $\left(\widetilde{U}_{h, m, j}^{d G}\right)_{j=0}^{r_{m}} \in\left(V_{h}\right)^{\underline{r}}$ such that there holds for all $\left(W_{m, i}\right)_{i=0}^{r_{m}} \in\left(V_{h}\right)^{\underline{r}}$,

$$
\sum_{i, j=0}^{r_{m}} C_{i j}\left(\widetilde{U}_{h, m, j}^{d G}, W_{m, i}\right)_{L^{2}(\Omega)}+\frac{k_{m}}{2} \sum_{i=0}^{r_{m}} \widetilde{a}\left(\widetilde{U}_{h, m, i}^{d G}, W_{m, i}\right)=\sum_{i=0}^{r_{m}} f_{m, i}\left(W_{m, i}\right),
$$

where, for $i, j=0,1, \ldots, r_{m}$,

$$
C_{i j}=\sigma_{i j} \sqrt{\left(i+\frac{1}{2}\right)\left(j+\frac{1}{2}\right)}, \quad \sigma_{i j}=\left\{\begin{array}{ll}
(-1)^{i+j} & \text { if } j>i \\
1 & \text { else }
\end{array},\right.
$$

and $f_{m, i}(v)=\phi_{i}(-1)\left(\widetilde{U}_{h, m-1}^{d G-}\left(t_{m-1}\right), v\right)$. 
From now on, for the sake of readability, we will drop the subscript $m$. Then, denoting by $\mathbf{M}$ and $\widetilde{\mathbf{A}}$ the mass and (compressed) stiffness matrix on $V^{h}=V^{L}$ with respect to $(\cdot, \cdot)$ and $\widetilde{a}(\cdot, \cdot)$, respectively, (3.23) takes the matrix form

$$
\mathbf{R} \underline{u}=\underline{f} \quad \text { with } \quad \mathbf{R}=\mathbf{C} \otimes \mathbf{M}+\frac{k}{2} \mathbf{I} \otimes \widetilde{\mathbf{A}},
$$

where $\underline{u}$ denotes the coefficient vector of $\widetilde{U}_{h, m}^{d G}=\left.\widetilde{U}_{h}^{d G}\right|_{I_{m}} \in \mathcal{P}_{r_{m}}\left(I_{m}, V_{h}\right)$.

4.2. Decoupling. In [38] it has been found that the system (4.3) of size $(r+1) N^{L}$ can be reduced to solving $r+1$ linear systems of size $N^{L}$. To this end, let $\mathbf{C}=\mathbf{Q} \mathbf{T Q} \mathbf{Q}^{\mathrm{H}}$ be the Schur decomposition of the $(r+1) \times(r+1)$ matrix $\mathbf{C}$ with a unitary matrix $\mathbf{Q}$ and an upper triangular matrix $\mathbf{T}$. Note that the diagonal of $\mathbf{T}$ contains the eigenvalues $\lambda_{1}, \lambda_{2}, \ldots, \lambda_{r+1}$ of C. Then, multiplying (4.3) by $\mathbf{Q}^{\mathrm{H}} \otimes \mathbf{I}$ from the left results in the linear system

$$
\left(\mathbf{T} \otimes \mathbf{M}+\frac{k}{2} \mathbf{I} \otimes \widetilde{\mathbf{A}}\right) \underline{w}=\underline{g} \quad \text { with } \quad \underline{w}=\left(\mathbf{Q}^{H} \otimes \mathbf{I}\right) \underline{u}, \quad \underline{g}=\left(\mathbf{Q}^{H} \otimes \mathbf{I}\right) \underline{f} .
$$

This system is block-upper-triangular. With $\underline{w}=\left(\underline{w}_{0}, \underline{w}_{1}, \ldots, \underline{w}_{r}\right), \underline{w}_{j} \in \mathbb{C}^{N^{L}}$, we obtain its solution by solving

$$
\left(\lambda_{j+1} \mathbf{M}+\frac{k}{2} \widetilde{\mathbf{A}}\right) \underline{w}_{j}=\underline{s}_{j}
$$

for $j=r, r-1, \ldots, 0$, where $\underline{s}_{j}=\underline{g}_{j}-\sum_{l=j+1}^{r} \mathbf{T}_{j+1, l+1} \mathbf{M} \underline{w}_{l}$.

4.3. Preconditioning and Iterative Solution. By (4.4), a dG-time step of order $r$ amounts to solving $r+1$ linear systems with a matrix of the form

$$
\mathbf{B}=\lambda \mathbf{M}+\frac{k}{2} \tilde{\mathbf{A}}
$$

Here, $\lambda$ is an eigenvalue of $\mathbf{C}$ in (4.2). Estimates on the eigenvalues of $\mathbf{C}$ have been established in [43].

For the preconditioning of the linear system (4.5), we define the matrix $\mathbf{S}$ and the scaled matrix $\widehat{\mathbf{B}} \in \mathbb{R}^{N^{L}} \times \mathbb{R}^{N^{L}}$ by

$$
\mathbf{S}=\left(\operatorname{Re}(\lambda) \mathbf{I}+\frac{k}{2} \mathbf{D}_{\mathbf{A}}\right)^{\frac{1}{2}}, \quad \widehat{\mathbf{B}}=\mathbf{S}^{-1} \mathbf{B S}^{-1}
$$

where $\mathbf{D}_{\mathbf{A}}$ is the diagonal matrix with entries $D_{(l, j),(l, j)}=2^{l \frac{\rho}{2}}$ corresponding to the basis functions $\psi_{j}^{l} \in V_{h}$; cf. (3.4).

The preconditioned linear equations corresponding to (4.4) are solved approximately with incomplete GMRES $\left(m_{0}\right)$ iteration (restarted every $m_{0} \geq 1$ iterations). There holds:

Theorem 4.1. Let the assumptions of Theorem 3.12 hold. Then, choosing the number and order of time steps such that $M=c|\log h|$ (with $c>0$ sufficiently large) and in each time step $n_{G}=\mathcal{O}(|\log h|)^{5}$ GMRES iterations, implies that

$$
\left\|u(T)-\widehat{U}_{h}^{d G}(T)\right\| \leq C T^{-\frac{p+1}{\rho}} h^{p+1},
$$

where $\widehat{U}_{h}^{d G}$ denotes the (perturbed) $d G$ approximation of the exact solution $u$ to (1.1)-(1.2) obtained by the incomplete GMRES method. 


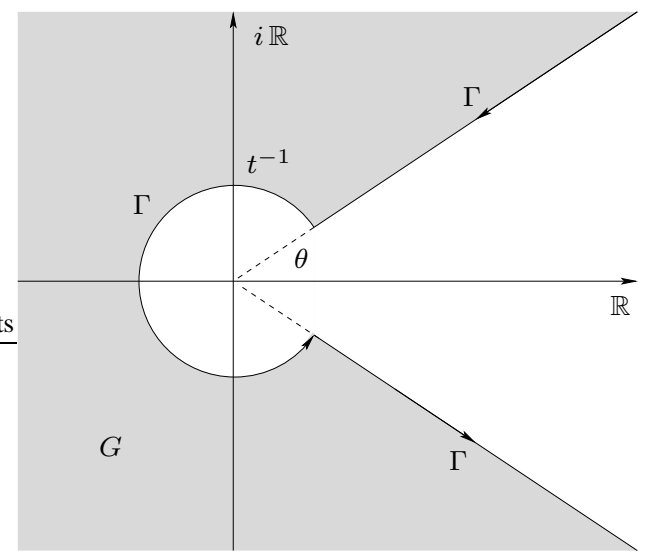

FIGURE 1

4.4. Complexity. Applying the matrix compression techniques from Section 3.1.2, the judicious combination of geometric mesh refinement and linear increase of polynomial degrees in the dG time-stepping scheme (Theorem 3.12), and an appropriate number of GMRES iterations (Theorem 4.1), results in an linear (up to some logarithmic terms) overall complexity of the fully discrete scheme (3.19) for the solution of the parabolic problem (1.1)-(1.2).

Theorem 4.2. Under the assumptions of Theorems 3.12 and 4.1, the fully discrete scheme (3.19) with $n_{G}=\mathcal{O}(|\log h|)^{5}$ GMRES iterations per time step yields $\widehat{U}_{h}^{d G}(T)$ in at most $\mathcal{O}\left(N(\log N)^{8}\right)$ operations, where $N=N^{L}=\operatorname{dim} V_{h}$ is the number of spatial degrees of freedom.

\section{Proofs of Theorems 3.1 And 3.6}

We will now establish the estimates (3.3) and (3.18). Without loss of generality, we will assume that $T=1$.

5.1. Proof of Theorem 3.1. We proceed in several steps.

Step 1. We first recall some well-known results from the theory of analytic semi-groups.

We note that, due to the continuity (2.1) and the coercivity (2.3) of the sesquilinear form $a$, the operator $\mathcal{A}$ is 'sectorial'. More precisely, we have the following results (see [22], for example):

Lemma 5.1. There exists an acute angle $\theta \in\left(0, \frac{\pi}{2}\right)$ such that

$$
G=\{z \in \mathbb{C}: \theta \leq|\arg z| \leq \pi\} \subset \rho(\mathcal{A})
$$

where $\rho(\mathcal{A})$ is the resolvent set of $\mathcal{A}$. Moreover, there are constants $M, \delta>0$ such that there holds

$$
\left\|(z-\mathcal{A})^{-1}\right\| \leq \frac{M}{|z|}, \quad \forall z \in G
$$

and

$$
|z|\|w\|^{2}+\|w\|_{V}^{2} \leq \delta\left|z\|w\|^{2}-a(w, w)\right|, \quad \forall w \in V, \forall z \in G
$$


The above result allows us to represent the solution $u(t)$ of (1.1)-(1.2) and the semidiscrete solution $u_{h}(t)$ of (3.1)-(3.2) in terms of a Dunford-Taylor integral representation:

$$
u(t)=e^{-t \mathcal{A}} u_{0}=\frac{1}{2 \pi i} \int_{\Gamma} e^{-t z}(z-\mathcal{A})^{-1} u_{0} d z
$$

and

$$
u_{h}(t)=e^{-t \mathcal{A}_{h}} P_{h} u_{0}=\frac{1}{2 \pi i} \int_{\Gamma} e^{-t z}\left(z-\mathcal{A}_{h}\right)^{-1} P_{h} u_{0} d z
$$

Here, $\mathcal{A}_{h}: V_{h} \rightarrow V_{h}$ is the finite element approximation of $\mathcal{A}$ defined by

$$
\left(\mathcal{A}_{h} \varphi_{h}, \psi_{h}\right)=a\left(\varphi_{h}, \psi_{h}\right), \quad \forall \varphi_{h}, \psi_{h} \in V_{h},
$$

and $\Gamma$ denotes the piecewise smooth path running around the sector $G$ (see Lemma 5.1) from $+\infty \cdot e^{i \theta}$ to $+\infty \cdot e^{-i \theta}$, i.e., $\Gamma=\Gamma_{1} \cup \Gamma_{2} \cup \Gamma_{3}$, with

$$
\begin{aligned}
& \Gamma_{1}=\left\{s e^{i \theta}: \infty \geq s \geq t^{-1}\right\}, \\
& \Gamma_{2}=\left\{t^{-1} e^{i s}: \theta \leq s \leq 2 \pi-\theta\right\}, \\
& \Gamma_{3}=\left\{s e^{-i \theta}: t^{-1} \leq s \leq \infty\right\} ;
\end{aligned}
$$

see Figure 1. We note that, since $V_{h} \subset V$, Lemma 5.1 also holds for the operator $\mathcal{A}_{h}$ (with the same constants $\theta, M, \delta)$; in particular, this implies that the term $\left(z-\mathcal{A}_{h}\right)^{-1}$ in (5.4) is well-defined (independently of $h$ ).

From (5.3), it follows that $\forall t>0, \forall l \in \mathbb{N}$, there exists a constant $C>0$ such that

$$
\left\|\mathcal{A}^{l} e^{-t \mathcal{A}}\right\| \leq C(l) t^{-l}
$$

Step 2. For the proof of Theorem 3.1, we need to estimate the term

$$
\left\|u(t)-u_{h}(t)\right\|=\left\|E_{h}(t) u_{0}\right\|
$$

appropriately, where the operator $E_{h}(t)$ is given by

$$
E_{h}(t)=e^{-t \mathcal{A}}-e^{-t \mathcal{A}_{h}} P_{h}=\frac{1}{2 \pi i} \int_{\Gamma} e^{-t z}\left((z-\mathcal{A})^{-1}-\left(z-\mathcal{A}_{h}\right)^{-1} P_{h}\right) d z
$$

The aim of this step is to prove the following

Lemma 5.2. For all $0<t \leq 1$, there holds

$$
\left\|E_{h}(t)\right\| \leq C \min \left\{1, h^{\rho} t^{-1}\right\}
$$

where $C>0$ is a constant independent of $t$, and

$$
\left\|E_{h}(t)\right\|=\sup _{v \in L^{2}(\Omega)} \frac{\left\|E_{h}(t) v\right\|}{\|v\|} .
$$

Proof. Let $z \in G$ with $|z| \geq t^{-1}$ be fixed, and define

$$
w=(z-\mathcal{A})^{-1} u_{0}, \quad w_{h}=\left(z-\mathcal{A}_{h}\right)^{-1} P_{h} u_{0} .
$$

Then, (5.1) implies that

$$
\|w\| \leq \frac{M}{|z|}\left\|u_{0}\right\|
$$

and due to $a(w, w)=z(w, w)-\left(u_{0}, w\right)$ and (2.3), it follows that

$$
\|w\|_{V}^{2} \leq \beta^{-1}\left(|z|\|w\|^{2}+\left\|u_{0}\right\|\|w\|\right) \leq C \frac{1}{|z|}\left\|u_{0}\right\|^{2} .
$$


Combining (5.9) and (5.10), results in

$$
|z|\|w\|+\sqrt{|z|}\|w\|_{V} \leq C\left\|u_{0}\right\| .
$$

Moreover, since $\mathcal{A} w=z w-u_{0}$, we obtain $\|\mathcal{A} w\| \leq C\left\|u_{0}\right\|$. Hence, using the fact that the graph norm of $\mathcal{A}$ is equivalent to the $\|\cdot\|_{\rho}$ norm, i.e. in particular,

$$
\|w\|_{\rho} \leq C(\|\mathcal{A} w\|+\|w\|)
$$

and noticing that, by (5.9), $\|w\| \leq M|z|^{-1}\left\|u_{0}\right\| \leq M t\left\|u_{0}\right\| \leq M\left\|u_{0}\right\|$, leads to

$$
\|w\|_{\rho} \leq C\left\|u_{0}\right\| \text {. }
$$

Summarizing, we have

$$
\|w\|_{\rho}+\sqrt{|z|}\|w\|_{V}+|z|\|w\| \leq C\left\|u_{0}\right\| .
$$

Referring to (5.2), noticing the Galerkin orthogonality

$$
z\left(w-w_{h}, v_{h}\right)-a\left(w-w_{h}, v_{h}\right)=0, \quad \forall v_{h} \in V_{h},
$$

and making use of the approximation property (3.7) of the operator $Q_{h}$, it follows that

$$
\begin{aligned}
|z|\left\|w-w_{h}\right\|^{2} & +\left\|w-w_{h}\right\|_{V}^{2} \\
& \leq \delta\left|z\left(w-w_{h}, w-w_{h}\right)-a\left(w-w_{h}, w-w_{h}\right)\right| \\
& =\delta\left|z\left(w-w_{h}, w-Q_{h} w\right)-a\left(w-w_{h}, w-Q_{h} w\right)\right| \\
& \leq C\left(|z|\left\|w-w_{h}\right\|\left\|w-Q_{h} w\right\|+\left\|w-w_{h}\right\|\left\|_{V}\right\| w-Q_{h} w \|_{V}\right) \\
& \leq C h^{\frac{\rho}{2}}\left(\sqrt{|z|}\left\|w-w_{h}\right\|+\left\|w-w_{h}\right\|_{V}\right)\left(\sqrt{|z|}\|w\|_{V}+\|w\|_{\rho}\right) .
\end{aligned}
$$

Applying the a priori estimate (5.13), yields

$$
\sqrt{|z|}\left\|w-w_{h}\right\|+\left\|w-w_{h}\right\|_{V} \leq C h^{\frac{\rho}{2}}\left\|u_{0}\right\| .
$$

Now, using the Nitsche trick, we prove that $\left\|w-w_{h}\right\| \leq C h^{\rho}\left\|u_{0}\right\|$. To this end, let $v=\left(\bar{z}-\mathcal{A}^{*}\right)^{-1}\left(w-w_{h}\right)$, where $\mathcal{A}^{*}$ is the adjoint operator of $\mathcal{A}$.

Repeating the argument for the derivation of (5.14) with $v, w-w_{h}$ instead of $w, u_{0}$, we obtain

$$
\begin{aligned}
\left\|w-w_{h}\right\|^{2} & =z\left(w-w_{h}, v\right)-a\left(w-w_{h}, v\right) \\
& =z\left(w-w_{h}, v-Q_{h} v\right)-a\left(w-w_{h}, v-Q_{h} v\right) \\
& \leq\left(\sqrt{|z|}\left\|v-Q_{h} v\right\|+\left\|v-Q_{h} v\right\|_{V}\right)\left(\sqrt{|z|}\left\|w-w_{h}\right\|+\left\|w-w_{h}\right\|_{V}\right) \\
& \leq C h^{\rho}\left\|w-w_{h}\right\|\left\|u_{0}\right\| .
\end{aligned}
$$

This implies

$$
\left\|w-w_{h}\right\| \leq C h^{\rho}\left\|u_{0}\right\| .
$$

Thus, recalling the Dunford-Taylor integral representation (5.6) and noticing that

$$
\int_{\Gamma}\left|e^{-t z}\right||d z| \leq C t^{-1}
$$

results in

$$
\left\|E_{h}(t) u_{0}\right\| \leq \frac{1}{2 \pi} \int_{\Gamma}\left|e^{-t z}\right|\left\|(z-\mathcal{A})^{-1} u_{0}-\left(z-\mathcal{A}_{h}\right)^{-1} P_{h} u_{0}\right\||d z| \leq C h^{\rho} t^{-1}\left\|u_{0}\right\|,
$$

which completes the proof.

Step 3. Next, we establish the following estimate: 
Lemma 5.3. For $t>0$, set $\bar{t}=\frac{t}{2}$ and $\bar{u}_{0}=e^{-\bar{t} \mathcal{A}} u_{0}=\frac{1}{2 \pi i} \int_{\Gamma} e^{-\bar{t} z}(z-\mathcal{A})^{-1} u_{0} d z$. Then, the estimate

$$
\left\|E_{h}(\bar{t}) \bar{u}_{0}\right\| \leq C \min \left\{1, h^{p+1} t^{-\frac{p+1}{\rho}}\right\}\left\|u_{0}\right\|
$$

holds true.

In order to prove this lemma, the ensuing result is required:

Lemma 5.4. Let $\bar{t}$ and $\bar{u}_{0}$ be as in the previous Lemma 5.3. Then, there exists a constant $C>0$ independent of $t$ and $h$ such that for all $z \in G$, there holds

$$
\sum_{k=0}^{s} \sqrt{|z|}^{s-k}\left\|(z-\mathcal{A})^{-1} \bar{u}_{0}\right\|_{k \frac{\rho}{2}} \leq C \sum_{l=0}^{s-2} \sqrt{|z|}^{s-2-l}\left\|\bar{u}_{0}\right\|_{l \frac{\rho}{2}}
$$

for all $s \geq 2$.

Proof. First of all, instead of (5.17), we prove that, for all $s \geq 2$, we have

$$
\sum_{k=0}^{s} \sqrt{|z|}^{s-k}\left\|\mathcal{A}^{\frac{k}{2}} \bar{w}\right\| \leq C \sum_{l=0}^{s-2} \sqrt{|z|}^{s-2-l}\left\|\mathcal{A}^{\frac{l}{2}} \bar{u}_{0}\right\|,
$$

where $\bar{w}=(z-\mathcal{A})^{-1} \bar{u}_{0}$.

This follows by induction with respect to $s$. For $s=2,(5.18)$ is equivalent to (5.13). Hence, assume that (5.18) is true for $s>2$. Then,

$$
\begin{aligned}
\sum_{k=0}^{s+1} \sqrt{|z|}^{s+1-k}\left\|\mathcal{A}^{\frac{k}{2}} \bar{w}\right\| & =\sqrt{|z|}^{s+1}\|\bar{w}\|+\sum_{k=0}^{s} \sqrt{|z|}^{s-k}\left\|\mathcal{A}^{\frac{k}{2}} \mathcal{A}^{\frac{1}{2}} \bar{w}\right\| \\
& \leq \sqrt{|z|}^{s+1}\|\bar{w}\|+C \sum_{l=0}^{s-2} \sqrt{|z|}^{s-2-l}\left\|\mathcal{A}^{\frac{l+1}{2}} \bar{u}_{0}\right\| \\
& =\sqrt{|z|}^{s+1}\|\bar{w}\|+C \sum_{l=1}^{s-1} \sqrt{|z|}^{s-1-l}\left\|\mathcal{A}^{\frac{l}{2}} \bar{u}_{0}\right\| \\
& \leq C \sum_{l=0}^{s-1} \sqrt{|z|}^{s-1-l}\left\|\mathcal{A}^{\frac{l}{2}} \bar{u}_{0}\right\|
\end{aligned}
$$

where in the last step, we have used (5.9) with $\bar{w}, \bar{u}_{0}$ instead of $w, u_{0}$.

Now, we use that the graph norm of $\mathcal{A}^{\frac{l}{2}}$ is equivalent to the $\|\cdot\|_{l \frac{\rho}{2}}$ norm, i.e., in particular,

$$
\begin{array}{ll}
\|\bar{w}\|_{k \frac{\rho}{2}} \cong\left\|\mathcal{A}^{\frac{k}{2}} \bar{w}\right\|+\|\bar{w}\|, & \forall 0 \leq k \leq s, \\
\left\|\bar{u}_{0}\right\|_{l \frac{\rho}{2}} \cong\left\|\mathcal{A}^{\frac{l}{2}} \bar{u}_{0}\right\|+\left\|\bar{u}_{0}\right\|, \quad \forall 0 \leq l \leq s-2 .
\end{array}
$$


Together with (5.9), this implies that

$$
\begin{aligned}
\sum_{k=0}^{s} \sqrt{|z|}^{s-k}\|\bar{w}\|_{\frac{k \rho}{2}} & \leq C \sum_{k=0}^{s} \sqrt{|z|}^{s-k}\left(\left\|\mathcal{A}^{\frac{k}{2}} \bar{w}\right\|+\|\bar{w}\|\right) \\
& \leq C \sum_{l=0}^{s-2} \sqrt{|z|}^{s-2-l}\left(\left\|\mathcal{A}^{\frac{l}{2}} \bar{u}_{0}\right\|+\left\|\bar{u}_{0}\right\|\right) \\
& \leq C \sum_{l=0}^{s-2} \sqrt{|z|}^{s-2-l}\left\|\bar{u}_{0}\right\|_{\frac{l \rho}{2}}
\end{aligned}
$$

Proof of Lemma 5.3. Applying (5.2) with

$$
\bar{w}=(z-\mathcal{A})^{-1} \bar{u}_{0}, \quad \bar{w}_{h}=\left(z-\mathcal{A}_{h}\right)^{-1} P_{h} \bar{u}_{0},
$$

and using the approximation property (3.7), implies that

$$
\begin{aligned}
|z|\left\|\bar{w}-\bar{w}_{h}\right\|^{2} & +\left\|\bar{w}-\bar{w}_{h}\right\|_{V}^{2} \\
& \leq \delta\left|z\left(\bar{w}-\bar{w}_{h}, \bar{w}-\bar{w}_{h}\right)-a\left(\bar{w}-\bar{w}_{h}, \bar{w}-\bar{w}_{h}\right)\right| \\
& =\delta\left|z\left(\bar{w}-\bar{w}_{h}, \bar{w}-Q_{h} \bar{w}\right)-a\left(\bar{w}-\bar{w}_{h}, \bar{w}-Q_{h} \bar{w}\right)\right| \\
& \leq \delta\left(|z|\left\|\bar{w}-\bar{w}_{h}\right\|\left\|\bar{w}-Q_{h} \bar{w}\right\|+\left\|\bar{w}-\bar{w}_{h}\right\|_{V}\left\|\bar{w}-Q_{h} \bar{w}\right\|_{V}\right) \\
& \leq C h^{\alpha}\left(\sqrt{|z|}|| \bar{w}-\bar{w}_{h}\|+\| \bar{w}-\bar{w}_{h} \|_{V}\right)\left(\sqrt{|z|}\|\bar{w}\|_{\alpha}+\|\bar{w}\|_{\alpha+\frac{\rho}{2}}\right),
\end{aligned}
$$

for all $\frac{\rho}{2} \leq \alpha \leq p+1-\frac{\rho}{2}$.

Consequently, for all $\frac{\rho}{2} \leq \alpha \leq p+1-\frac{\rho}{2}$, there holds

$$
\sqrt{|z|}\left\|\bar{w}-\bar{w}_{h}\right\|+\left\|\bar{w}-\bar{w}_{h}\right\|_{V} \leq C h^{\alpha}\left(\sqrt{|z|}\|\bar{w}\|_{\alpha}+\|\bar{w}\|_{\alpha+\frac{\rho}{2}}\right) .
$$

By Nitsche's trick, the $L^{2}$ norm error can be improved to

$$
\left\|\bar{w}-\bar{w}_{h}\right\| \leq C h^{\alpha+\frac{\rho}{2}}\left(\sqrt{|z|}\|\bar{w}\|_{\alpha}+\|\bar{w}\|_{\alpha+\frac{\rho}{2}}\right) .
$$

Indeed, by defining $\bar{v}=\left(\bar{z}-\mathcal{A}^{*}\right)^{-1}\left(\bar{w}-\bar{w}_{h}\right)$ and $\bar{v}_{h}=\left(\bar{z}-\mathcal{A}_{h}^{*}\right)^{-1} P_{h}\left(\bar{w}-\bar{w}_{h}\right)$, we obtain

$$
\begin{aligned}
\left\|\bar{w}-\bar{w}_{h}\right\|^{2} & =\left|z\left(\bar{w}-\bar{w}_{h}, \bar{v}\right)-a\left(\bar{w}-\bar{w}_{h}, \bar{v}\right)\right| \\
& =\left|z\left(\bar{w}-\bar{w}_{h}, \bar{v}-\bar{v}_{h}\right)-a\left(\bar{w}-\bar{w}_{h}, \bar{v}-\bar{v}_{h}\right)\right| \\
& \leq\left(\sqrt{|z|}\left\|\bar{w}-\bar{w}_{h}\right\|+\left\|\bar{w}-\bar{w}_{h}\right\|_{V}\right)\left(\sqrt{|z|}\left\|\bar{v}-\bar{v}_{h}\right\|+\left\|\bar{v}-\bar{v}_{h}\right\|_{V}\right) \\
& \leq C h^{\alpha+\frac{\rho}{2}}\left(\sqrt{|z|}\|\bar{w}\|_{\alpha}+\|\bar{w}\|_{\alpha+\frac{\rho}{2}}\right)\left\|\bar{w}-\bar{w}_{h}\right\|,
\end{aligned}
$$

where, in the last step, we have used (5.21) and (5.14) with $\bar{v}, \bar{v}_{h}, \bar{w}-\bar{w}_{h}$ instead of $w$, $w_{h}, u_{0}$. Therefore,

$$
\left\|\bar{w}-\bar{w}_{h}\right\| \leq C h^{\alpha+\frac{\rho}{2}}\left(\sqrt{|z|}\|\bar{w}\|_{\alpha}+\|\bar{w}\|_{\alpha+\frac{\rho}{2}}\right) .
$$


Inserting (5.22) into the Dunford-Taylor integral representation (5.6), yields

$$
\begin{aligned}
\| E_{h}\left(\frac{t}{2}\right) & e^{-\frac{t}{2} \mathcal{A}} u_{0} \| \\
& =\left\|E_{h}(\bar{t}) \bar{u}_{0}\right\| \\
& =\frac{1}{2 \pi}\left|\int_{\Gamma} e^{-\frac{t}{2} z}\left((z-\mathcal{A})^{-1} \bar{u}_{0}-\left(z-\mathcal{A}_{h}\right)^{-1} P_{h} \bar{u}_{0}\right) d z\right| \\
& \leq \frac{1}{2 \pi} \int_{\Gamma}\left|e^{-\frac{t}{2} z}\right|\left\|\bar{w}-\bar{w}_{h}\right\||d z| \\
& \leq C h^{\alpha+\frac{\rho}{2}} \int_{\Gamma}\left|e^{-\frac{t}{2} z}\right|\left(\sqrt{|z|}\|\bar{w}\|_{\alpha}+\|\bar{w}\|_{\alpha+\frac{\rho}{2}}\right)|d z| .
\end{aligned}
$$

Selecting $\alpha=p+1-\frac{\rho}{2}$ in (5.23), leads to

$$
\left\|E_{h}\left(\frac{t}{2}\right) e^{-\frac{t}{2} \mathcal{A}} u_{0}\right\| \leq C h^{p+1} \int_{\Gamma}\left|e^{-\frac{t}{2} z}\right|\left(\sqrt{|z|}\|\bar{w}\|_{p+1-\frac{\rho}{2}}+\|\bar{w}\|_{p+1}\right)|d z| .
$$

Let $s \in \mathbb{N}$ and $\theta \in[0,1)$ be such that

and

$$
s \frac{\rho}{2} \leq p+1<(s+1) \frac{\rho}{2},
$$

$$
p+1=(1-\theta) s \frac{\rho}{2}+\theta(s+1) \frac{\rho}{2}=(\theta+s) \frac{\rho}{2} .
$$

Then, by (5.17), we have

$$
\sqrt{|z|}\|\bar{w}\|_{s \frac{\rho}{2}}+\|\bar{w}\|_{(s+1) \frac{\rho}{2}} \leq C \sum_{l=0}^{s-1} \sqrt{|z|}^{s-1-l}\left\|\bar{u}_{0}\right\|_{l \frac{\rho}{2}},
$$

which, by interpolation, implies that

$$
\sqrt{|z|}^{1-\theta}\|\bar{w}\|_{p+1} \leq C \sum_{l=0}^{s-1} \sqrt{|z|}^{s-1-l}\left\|\bar{u}_{0}\right\|_{l \frac{\rho}{2}} .
$$

Analogously, we obtain

$$
\sqrt{|z|}^{2-\theta}\|\bar{w}\|_{p+1-\frac{\rho}{2}} \leq C \sum_{l=0}^{s-1} \sqrt{|z|}^{s-1-l}\left\|\bar{u}_{0}\right\|_{l \frac{\rho}{2}} .
$$

Thus, using the above estimates and (5.20), (5.24) transforms to

$$
\begin{aligned}
& \left\|E_{h}\left(\frac{t}{2}\right) e^{-\frac{t}{2} \mathcal{A}} u_{0}\right\| \\
& \quad \leq\left. C h^{p+1} \int_{\Gamma}\left|e^{-\frac{t}{2} z}\right| \sum_{l=0}^{s-1} \sqrt{|z|}\right|^{s-2-l+\theta}\left(\left\|\mathcal{A}^{\frac{l}{2}} e^{-\frac{t}{2} \mathcal{A}} u_{0}\right\|+\left\|e^{-\frac{t}{2} \mathcal{A}} u_{0}\right\|\right)|d z| \\
& \quad \leq C h^{p+1} t^{-1} \sum_{l=0}^{s-1} t^{-\frac{s-2-l+\theta}{2}}\left(t^{-\frac{l}{2}}+1\right)\left\|u_{0}\right\| \\
& \quad \leq C h^{p+1} t^{-\frac{s+\theta}{2}}\left\|u_{0}\right\| .
\end{aligned}
$$

By the choice of $\theta$ in (5.25), it follows that

$$
\left\|E_{h}\left(\frac{t}{2}\right) e^{-\frac{t}{2} \mathcal{A}} u_{0}\right\| \leq C h^{p+1} t^{-\frac{p+1}{\rho}}\left\|u_{0}\right\| .
$$


Step 4. We are now in a position to prove Theorem 3.1. Note that there holds the decomposition

$$
E_{h}(t)=-E_{h}\left(\frac{t}{2}\right) E_{h}\left(\frac{t}{2}\right)+E_{h}\left(\frac{t}{2}\right) e^{-\frac{t}{2} \mathcal{A}}+e^{-\frac{t}{2} \mathcal{A}} E_{h}\left(\frac{t}{2}\right)
$$

and thus

$$
\left\|E_{h}(t) u_{0}\right\| \leq\left\|E_{h}\left(\frac{t}{2}\right) E_{h}\left(\frac{t}{2}\right) u_{0}\right\|+\left\|E_{h}\left(\frac{t}{2}\right) e^{-\frac{t}{2} \mathcal{A}} u_{0}\right\|+\left\|e^{-\frac{t}{2} \mathcal{A}} E_{h}\left(\frac{t}{2}\right) u_{0}\right\| .
$$

The first and the second term on the right-hand side of the above inequality are estimated by Lemma 5.2 and Lemma 5.3. A bound for the last term can be obtained similarly as for the second term using a duality argument. This yields

$$
\left\|E_{h}(t) u_{0}\right\| \leq C \min \left\{1, h^{\rho} t^{-1}\right\}\left\|E_{h}\left(\frac{t}{2}\right) u_{0}\right\|+C \min \left\{1, h^{p+1} t^{-\frac{p+1}{\rho}}\right\}\left\|u_{0}\right\|,
$$

which, applied recursively, results in

$$
\left\|E_{h}(t) u_{0}\right\| \leq C \min \left\{1, h^{p+1} t^{-\frac{p+1}{\rho}}\right\}\left\|u_{0}\right\| .
$$

This completes the proof of Theorem 3.1.

5.2. Proof of Theorem 3.6. Again, we split the proof into several parts.

Step 1. Since the perturbed sesquilinear form $\widetilde{a}$ is continuous and coercive on the space $V_{h}$ (cf. (3.10), (3.11)), there holds the inequality, analogous to (5.2),

$$
|z|\left\|w_{h}\right\|^{2}+\left\|w_{h}\right\|_{V}^{2} \leq \widetilde{\delta}\left|z\left\|w_{h}\right\|^{2}-\widetilde{a}\left(w_{h}, w_{h}\right)\right|, \quad \forall w_{h} \in V_{h}, \forall z \in \widetilde{G}_{h},
$$

where $\widetilde{\delta}>0$ is a constant independent of the mesh size $h$, and $\widetilde{G}_{h}$ is a sector in $\mathbb{C}$. Without loss of generality, we can assume that $\widetilde{G}_{h} \subset G$, where $G$ is the sector from Lemma 5.1. As in (5.4), the solution $\widetilde{u}_{h}(t)$ of the (perturbed) semi-discrete problem (3.16)-(3.17) can be represented in the form

$$
\widetilde{u}_{h}(t)=e^{-t \widetilde{\mathcal{A}}_{h}} P_{h} u_{0}=\frac{1}{2 \pi i} \int_{\Gamma} e^{-t z}\left(z-\widetilde{\mathcal{A}}_{h}\right)^{-1} P_{h} u_{0} d z,
$$

where the operator $\widetilde{\mathcal{A}}_{h}: V_{h} \rightarrow V_{h}$ is defined by

$$
\left(\widetilde{\mathcal{A}}_{h} \varphi_{h}, \psi_{h}\right)=\widetilde{a}\left(\varphi_{h}, \psi_{h}\right), \quad \forall \varphi_{h}, \psi_{h} \in V_{h} .
$$

For the proof of Theorem 3.1, we will study the perturbation operator

$$
\widetilde{E}_{h}(t)=\frac{1}{2 \pi i} \int_{\Gamma} e^{-t z}\left(\left(z-\mathcal{A}_{h}\right)^{-1} P_{h}-\left(z-\widetilde{\mathcal{A}}_{h}\right)^{-1} P_{h}\right) d z,
$$

where $\Gamma$ is defined as in the previous Section 5.1; see also Figure 1. The aim of the following Steps 2 and 3 is to prove that, for $t>0$, there holds

$$
\begin{aligned}
\left\|\widetilde{E}_{h}(t) u_{0}\right\| & \leq C \frac{h^{\rho}}{t}\left\|u_{0}\right\|, \\
\left\|\widetilde{E}_{h}\left(\frac{t}{2}\right) e^{-\frac{t}{2} \mathcal{A}} u_{0}\right\| & \leq C h^{p+1} t^{-\frac{p+1}{\rho}}\left\|u_{0}\right\| .
\end{aligned}
$$


Step 2. We prove (5.33). To do so, define $\widetilde{w}_{h}=\left(z-\widetilde{\mathcal{A}}_{h}\right)^{-1} P_{h} u_{0}$, and let $w$ and $w_{h}$ be given as in (5.8). Furthermore, let $v_{h}=w_{h}-\widetilde{w}_{h}$. Then, recalling (5.32) and using the properties (3.14) and (3.7) of the operator $Q_{h}$, leads to

$$
\begin{aligned}
|z|\left\|w_{h}-\widetilde{w}_{h}\right\|^{2}+ & \left\|w_{h}-\widetilde{w}_{h}\right\|_{V}^{2} \\
\leq & \widetilde{\delta}\left|z\left(w_{h}-\widetilde{w}_{h}, w_{h}-\widetilde{w}_{h}\right)-\widetilde{a}\left(w_{h}-\widetilde{w}_{h}, w_{h}-\widetilde{w}_{h}\right)\right| \\
= & \widetilde{\delta}\left|a\left(w_{h}, w_{h}-\widetilde{w}_{h}\right)-\widetilde{a}\left(w_{h}, w_{h}-\widetilde{w}_{h}\right)\right| \\
\leq & \widetilde{\delta}\left|a\left(w_{h}-Q_{h} w, w_{h}-\widetilde{w}_{h}\right)-\widetilde{a}\left(w_{h}-Q_{h} w, w_{h}-\widetilde{w}_{h}\right)\right| \\
& +\widetilde{\delta}\left|a\left(Q_{h} w, w_{h}-\widetilde{w}_{h}\right)-\widetilde{a}\left(Q_{h} w, w_{h}-\widetilde{w}_{h}\right)\right| \\
\leq & C\left\|w_{h}-Q_{h} w\right\|_{V}\left\|w_{h}-\widetilde{w}_{h}\right\|_{V}+C h^{\frac{\rho}{2}}\|w\|_{\rho}\left\|w_{h}-\widetilde{w}_{h}\right\|_{V} \\
\leq & C\left(\left\|w-w_{h}\right\|_{V}+\left\|w-Q_{h} w\right\|_{V}+h^{\frac{\rho}{2}}\|w\|_{\rho}\right)\left\|w_{h}-\widetilde{w}_{h}\right\|_{V} \\
\leq & C\left(\left\|w-w_{h}\right\|_{V}+h^{\frac{\rho}{2}}\|w\|_{\rho}\right)\left\|w_{h}-\widetilde{w}_{h}\right\|_{V},
\end{aligned}
$$

which, by (5.12) and (5.14), yields the bound

$$
\sqrt{|z|}\left\|w_{h}-\widetilde{w}_{h}\right\|+\left\|w_{h}-\widetilde{w}_{h}\right\|_{V} \leq C h^{\frac{\rho}{2}}\left\|u_{0}\right\| .
$$

Next, let $v=\left(\bar{z}-\mathcal{A}^{*}\right)^{-1}\left(w_{h}-\widetilde{w}_{h}\right)$. Then,

$$
\begin{aligned}
\left\|w_{h}-\widetilde{w}_{h}\right\|^{2}= & z\left(w_{h}-\widetilde{w}_{h}, v\right)-a\left(w_{h}-\widetilde{w}_{h}, v\right) \\
= & z\left(w_{h}-\widetilde{w}_{h}, v-Q_{h} v\right)-a\left(w_{h}-\widetilde{w}_{h}, v-Q_{h} v\right) \\
& +z\left(w_{h}-\widetilde{w}_{h}, Q_{h} v\right)-a\left(w_{h}-\widetilde{w}_{h}, Q_{h} v\right) \\
= & z\left(w_{h}-\widetilde{w}_{h}, v-Q_{h} v\right)-a\left(w_{h}-\widetilde{w}_{h}, v-Q_{h} v\right) \\
& +a\left(\widetilde{w}_{h}-Q_{h} w, Q_{h} v\right)-\widetilde{a}\left(\widetilde{w}_{h}-Q_{h} w, Q_{h} v\right) \\
& +a\left(Q_{h} w, Q_{h} v\right)-\widetilde{a}\left(Q_{h} w, Q_{h} v\right) \\
\leq & |z|\left\|w_{h}-\widetilde{w}_{h}\right\|\left\|v-Q_{h} v\right\|+\left\|w_{h}-\widetilde{w}_{h}\right\|_{V}\left\|v-Q_{h} v\right\|_{V} \\
& +\left|a\left(Q_{h}\left(\widetilde{w}_{h}-w\right), Q_{h} v\right)-\widetilde{a}\left(Q_{h}\left(\widetilde{w}_{h}-w\right), Q_{h} v\right)\right| \\
& +\left|a\left(Q_{h} w, Q_{h} v\right)-\widetilde{a}\left(Q_{h} w, Q_{h} v\right)\right| .
\end{aligned}
$$

Now, we employ (3.15), (3.7) and (5.12) to obtain

$$
\begin{aligned}
\| w_{h}- & \widetilde{w}_{h} \|^{2} \\
\leq & \left(\sqrt{|z|}\left\|w_{h}-\widetilde{w}_{h}\right\|+\left\|w_{h}-\widetilde{w}_{h}\right\|_{V}\right)\left(\sqrt{|z|}\left\|v-Q_{h} v\right\|+\left\|v-Q_{h} v\right\|_{V}\right) \\
& +C h^{\frac{\rho}{2}}\left\|\widetilde{w}_{h}-w\right\|_{V}\|v\|_{\rho}+C h^{\rho}\|w\|_{\rho}\|v\|_{\rho} \\
\leq & C h^{\frac{\rho}{2}}\left(\sqrt{|z|}\left\|w_{h}-\widetilde{w}_{h}\right\|+\left\|w_{h}-\widetilde{w}_{h}\right\|_{V}\right)\left(\sqrt{|z|}\|v\|_{V}+\|v\|_{\rho}\right) \\
& +C h^{\frac{\rho}{2}}\left\|\widetilde{w}_{h}-w\right\|_{V}\|v\|_{\rho}+C h^{\rho}\left\|u_{0}\right\|\|v\|_{\rho} \\
\leq & C h^{\frac{\rho}{2}}\left(\sqrt{|z|}\left\|w_{h}-\widetilde{w}_{h}\right\|+\left\|w_{h}-\widetilde{w}_{h}\right\|_{V}+C h^{\frac{\rho}{2}}\left\|u_{0}\right\|\right)\left(\sqrt{|z|}\|v\|_{V}+\|v\|_{\rho}\right),
\end{aligned}
$$

where, in the last step, we have used the fact that, by (5.14), there holds

$$
\left\|\widetilde{w}_{h}-w\right\|_{V} \leq\left\|\widetilde{w}_{h}-w_{h}\right\|_{V}+\left\|w_{h}-w\right\|_{V} \leq\left\|\widetilde{w}_{h}-w_{h}\right\|_{V}+C h^{\frac{\rho}{2}}\left\|u_{0}\right\| .
$$


Hence, inserting (5.36) into (5.38) and applying (5.13) with $v$ and $w_{h}-\widetilde{w}_{h}$ instead of $w$ and $u_{0}$, respectively, it follows that

$$
\left\|w_{h}-\widetilde{w}_{h}\right\| \leq C h^{\rho}\left\|u_{0}\right\| .
$$

Step 3. Let $\widetilde{\bar{w}}_{h}=\left(z-\widetilde{\mathcal{A}}_{h}\right)^{-1} P_{h} \bar{u}_{0}$. In order to prove (5.34), we proceed as in (5.35) and use (3.14), (3.7) with $\frac{\rho}{2} \leq \alpha \leq p+1-\frac{\rho}{2}$, thereby obtaining

$$
\begin{aligned}
|z|\left\|\bar{w}_{h}-\widetilde{\bar{w}}_{h}\right\|^{2}+ & \left\|\bar{w}_{h}-\widetilde{\bar{w}}_{h}\right\|_{V}^{2} \\
\leq & \tilde{\delta}\left|a\left(\bar{w}_{h}-Q_{h} \bar{w}, \bar{w}_{h}-\widetilde{\bar{w}}_{h}\right)-\widetilde{a}\left(\bar{w}_{h}-Q_{h} \bar{w}, \bar{w}_{h}-\widetilde{\bar{w}}_{h}\right)\right| \\
& +\widetilde{\delta}\left|a\left(Q_{h} \bar{w}, \bar{w}_{h}-\widetilde{w}_{h}\right)-\widetilde{a}\left(Q_{h} \bar{w}, \bar{w}_{h}-\widetilde{\bar{w}}_{h}\right)\right| \\
\leq & C\left\|\bar{w}_{h}-Q_{h} \bar{w}\right\|_{V}\left\|\bar{w}_{h}-\widetilde{\bar{w}}_{h}\right\|_{V}+C h^{\alpha}\|\bar{w}\|_{\alpha+\frac{\rho}{2}}\left\|\bar{w}_{h}-\widetilde{w}_{h}\right\|_{V} \\
\leq & C\left(\left\|\bar{w}_{h}-\bar{w}\right\|_{V}+\left\|\bar{w}-Q_{h} \bar{w}\right\|_{V}+h^{\alpha}\|\bar{w}\|_{\alpha+\frac{\rho}{2}}\right)\left\|\bar{w}_{h}-\widetilde{\bar{w}}_{h}\right\|_{V} \\
\leq & C h^{\alpha}\left(\sqrt{|z|}\|\bar{w}\|_{\alpha}+\|\bar{w}\|_{\alpha+\frac{\rho}{2}}\right)\left\|\bar{w}_{h}-\widetilde{\bar{w}}_{h}\right\|_{V},
\end{aligned}
$$

where we have used (5.21) in the last inequality. Therefore, for all $\frac{\rho}{2} \leq \alpha \leq p+1-\frac{\rho}{2}$, there holds

$$
\sqrt{|z|} \mid\left\|\bar{w}_{h}-\widetilde{w}_{h}\right\|+\left\|\bar{w}_{h}-\widetilde{w}_{h}\right\|_{V} \leq C h^{\alpha}\left(\sqrt{|z|}\|\bar{w}\|_{\alpha}+\|\bar{w}\|_{\alpha+\frac{\rho}{2}}\right) .
$$

The estimate for the $L^{2}$ norm can be improved to

$$
\left\|\bar{w}_{h}-\widetilde{\bar{w}}_{h}\right\| \leq C h^{\alpha+\frac{\rho}{2}}\left(\sqrt{|z|}\|\bar{w}\|_{\alpha}+\|\bar{w}\|_{\alpha+\frac{\rho}{2}}\right),
$$

for all $\frac{\rho}{2} \leq \alpha \leq p+1-\frac{\rho}{2}$. Indeed, with $\bar{v}=\left(\bar{z}-A^{*}\right)^{-1}\left(\bar{w}_{h}-\widetilde{\bar{w}}_{h}\right)$, it holds, similar to (5.37),

$$
\begin{aligned}
\left\|\bar{w}_{h}-\widetilde{\bar{w}}_{h}\right\|^{2}= & z\left(\bar{w}_{h}-\widetilde{\bar{w}}_{h}, \bar{v}-Q_{h} \bar{v}\right)-a\left(\bar{w}_{h}-\widetilde{\bar{w}}_{h}, \bar{v}-Q_{h} \bar{v}\right) \\
& +a\left(\widetilde{\bar{w}}_{h}-Q_{h} \bar{w}, Q_{h} \bar{v}\right)-\widetilde{a}\left(\widetilde{\bar{w}}_{h}-Q_{h} \bar{w}, Q_{h} \bar{v}\right) \\
& +a\left(Q_{h} \bar{w}, Q_{h} \bar{v}\right)-\widetilde{a}\left(Q_{h} \bar{w}, Q_{h} \bar{v}\right) .
\end{aligned}
$$

Hence, as in (5.38), by applying (3.15), (3.7) with $\frac{\rho}{2} \leq \alpha \leq p+1-\frac{\rho}{2}$, and inserting (5.39), we obtain

$$
\begin{aligned}
\left\|\bar{w}_{h}-\widetilde{w}_{h}\right\|^{2} & \leq C h^{\alpha+\frac{\rho}{2}}\left(\sqrt{|z|}\|\bar{w}\|_{\alpha}+\|\bar{w}\|_{\alpha+\frac{\rho}{2}}\right)\left(\sqrt{|z|}\|\bar{v}\|_{V}+\|\bar{v}\|_{\rho}\right) \\
& \leq C h^{\alpha+\frac{\rho}{2}}\left(\sqrt{|z|}\|\bar{w}\|_{\alpha}+\|\bar{w}\|_{\alpha+\frac{\rho}{2}}\right)\left\|\bar{w}_{h}-\widetilde{w}_{h}\right\|
\end{aligned}
$$

where in the latter step, we have used (5.13) with $\bar{v}$ and $\bar{w}_{h}-\widetilde{\bar{w}}_{h}$ instead of $w$ and $u_{0}$. Putting $\alpha=p+1-\frac{\rho}{2}$ and referring to (5.40), results in

$$
\left\|\widetilde{E}_{h}\left(\frac{t}{2}\right) e^{-\frac{t}{2} \mathcal{A}} u_{0}\right\| \leq C h^{p+1} \int_{\Gamma}\left|e^{-t z}\right|\left(\sqrt{|z|}\|\bar{w}\|_{p+1-\frac{\rho}{2}}+\|\bar{w}\|_{p+1}\right)|d z| .
$$

Proceeding as in (5.27), we finally get

$$
\left\|\widetilde{E}_{h}\left(\frac{t}{2}\right) e^{-\frac{t}{2} A} u_{0}\right\| \leq C h^{p+1} t^{-\frac{p+1}{\rho}}\left\|u_{0}\right\| .
$$


Step 4. We have the decomposition

$$
\begin{aligned}
\widetilde{E}_{h}(t)= & -\widetilde{E}_{h}\left(\frac{t}{2}\right) \widetilde{E}_{h}\left(\frac{t}{2}\right)+\widetilde{E}_{h}\left(\frac{t}{2}\right) e^{-\frac{t}{2} \mathcal{A}_{h}} P_{h}+e^{-\frac{t}{2} \mathcal{A}_{h}} \widetilde{E}_{h}\left(\frac{t}{2}\right) \\
= & -\widetilde{E}_{h}\left(\frac{t}{2}\right) \widetilde{E}_{h}\left(\frac{t}{2}\right)+\widetilde{E}_{h}\left(\frac{t}{2}\right) e^{-\frac{t}{2} \mathcal{A}}-\widetilde{E}_{h}\left(\frac{t}{2}\right) E_{h}\left(\frac{t}{2}\right) \\
& -E_{h}\left(\frac{t}{2}\right) \widetilde{E}_{h}\left(\frac{t}{2}\right)+e^{-\frac{t}{2} \mathcal{A}} \widetilde{E}_{h}\left(\frac{t}{2}\right) .
\end{aligned}
$$

Inserting (5.33)-(5.34) into (5.41), applying Lemma 5.3, and using a duality argument, leads to the estimate

$$
\left\|\widetilde{E}_{h}(t) u_{0}\right\| \leq C \max \left\{1, h^{\rho} t^{-1}\right\}\left\|\widetilde{E}_{h}\left(\frac{t}{2}\right) u_{0}\right\|+C \min \left\{1, h^{p+1} t^{-\frac{p+1}{\rho}}\right\}\left\|u_{0}\right\|,
$$

which can be iterated to obtain

$$
\left\|\widetilde{E}_{h}(t) u_{0}\right\| \leq C \min \left\{1, h^{p+1} t^{-\frac{p+1}{\rho}}\right\}\left\|u_{0}\right\| .
$$

Now, the proof of Theorem 3.6 follows directly from the fact that

$$
\left\|u(t)-\widetilde{u}_{h}(t)\right\|=\left\|E_{h}(t) u_{0}+\widetilde{E}_{h}(t) u_{0}\right\| \leq\left\|E_{h}(t) u_{0}\right\|+\left\|\widetilde{E}_{h}(t) u_{0}\right\|,
$$

and from (5.31), (5.43).

\section{APPLICATIONS}

The aim of the following Section is to show that the linear complexity algorithm for the numerical solution of (1.1)-(1.2) presented in this paper is applicable to certain parabolic integro-differential equations arising in mathematical finance in the problem of option pricing.

6.1. Option Pricing. For risky assets whose log-returns are modeled by Feller price processes $X_{t}$ (see $[4,36]$ ) instead of the standard Wiener process $W_{t}$ in the Black-Scholes model, the infinitesimal generator $\mathcal{L}^{X}$ of the transition semi-group of $X_{t}$ is an integrodifferential operator.

Specifically, we consider the problem of pricing a European option with asset price $S_{t}$ given by the exponential law

$$
S_{t}=S_{0} e^{X_{t}}
$$

with $X_{t}$ a Lévy process.

For a given pay-off $f(S)$, the value of the European option $V(t, S)$ at $S=S_{t}$ with maturity $T>0$ is expressed by the following discounted conditional expectation,

$$
V(t, S)=\mathbf{E}^{\mathbb{Q}}\left[e^{-r(T-t)} f\left(S_{T}\right) \mid S_{t}=S\right],
$$

where $\mathbb{Q}$ is a martingale measure equivalent to the historical measure (see [7, 26, 29] for further details) and $r$ denotes the riskless mean-rate of return of $S_{t}$ under the measure $\mathbb{Q}$.

Itô's formula for semi-martingales and the principle of no-arbitrage imply that $V$ defined in (6.1) solves the parabolic integro-differential equation (cf. [35], see also [13, Proposition 1])

$$
\begin{aligned}
& \frac{\partial V}{\partial t}(t, S)+\frac{\sigma^{2}}{2} S^{2} \frac{\partial^{2} V}{\partial S^{2}}(t, S)+r S \frac{\partial V}{\partial S}(t, S)-r V(t, S) \\
& \quad+\int_{\mathbb{R}}\left(V\left(t, S e^{y}\right)-V(t, S)-S\left(e^{y}-1\right) \frac{\partial V}{\partial S}(t, S)\right) \nu_{\mathbb{Q}}(d y)=0
\end{aligned}
$$


in $J \times(0, \infty)$, and

$$
V(T, S)=f(S)
$$

in $(0, \infty)$. Here, by $\sigma \geq 0$, we denote the diffusion component of the Lévy process, i.e., $X_{t}$ can be uniquely decomposed as $X_{t}=\sigma W_{t}+Y_{t}$ with $W_{t}$ being a standard Brownian motion and $Y_{t}$ a quadratic pure jump process independent of $W_{t}$. Furthermore, $\nu_{\mathbb{Q}}(d y)$ represents the Lévy measure satisfying

$$
\int_{\mathbb{R}} \min \left(1, y^{2}\right) \nu_{\mathbb{Q}}(d y)<\infty .
$$

Henceforth, we assume that $\nu_{\mathbb{Q}}(d y)$ has a density $k_{\mathbb{Q}}$, i.e., $\nu_{\mathbb{Q}}(d y)=k_{\mathbb{Q}}(y) d y$. The Lévy density $k_{\mathbb{Q}}(y)$ describes the activity of jumps of size $y$ in $X_{t}$ in the sense that jumps of sizes in a set $A \subset \mathbb{R}$ occur according to a Poisson process of parameter $\int_{A} k_{\mathbb{Q}}(y) d y$. We say that the Lévy process $X_{t}$ is of finite activity if $k_{\mathbb{Q}}(y)$ is integrable; otherwise $X_{t}$ is said to be of infinite activity.

We drop the subscript $\mathbb{Q}$ in (6.2) and switch to logarithmic price $x=\log (S)$ and to time to maturity $t \rightarrow T-t$, thereby obtaining

$$
\begin{aligned}
\frac{\partial u}{\partial t}(t, x)+\mathcal{L}^{X}[u](t, x) & =0 & & \text { in } J \times \mathbb{R} \\
u(0, x) & =g(x) & & \text { in } \mathbb{R},
\end{aligned}
$$

where $g(x):=f\left(e^{x}\right)$ and $\mathcal{L}^{X}=\mathcal{L}^{\mathrm{BS}}+\mathcal{L}^{\text {jump }}$ with

$$
\begin{aligned}
\mathcal{L}^{\mathrm{BS}}[v] & :=-\frac{\sigma^{2}}{2} v^{\prime \prime}+\left(\frac{\sigma^{2}}{2}-r\right) v^{\prime}+r v, \\
\mathcal{L}^{\mathrm{jump}}[v](x) & :=-\int_{\mathbb{R}}\left\{v(x+y)-v(x)-\left(e^{y}-1\right) v^{\prime}(x)\right\} k(y) d y .
\end{aligned}
$$

Lévy processes of practical importance in the modeling of asset returns (examples are given below) typically satisfy some or all of the following assumptions (see also [33]):

(A1) Activity of small jumps: the characteristic function $\psi_{0}(u)$ of the pure jump part $Y_{t}$ of the Lévy process $X_{t}$ satisfies: there exist constants $c_{1}, C_{+}>0$ and $\alpha<2$ such that

$$
\left|\psi_{0}(\xi)-i c_{1} \xi\right| \leq C_{+}\left(1+|\xi|^{2}\right)^{\alpha / 2}, \quad \forall \xi \in \mathbb{R}
$$

(A2) Semi-heavy tails: there are constants $C>0, \eta_{-}>0$ and $\eta_{+}>1$ such that

$$
\forall|z|>1: \quad k(z) \leq C \begin{cases}e^{-\eta_{-}|z|} & \text { if } z<0 \\ e^{-\eta_{+}|z|} & \text { if } z>0 .\end{cases}
$$

(A3) Smoothness: for all $\beta \in \mathbb{N}_{0}$, there exists $C(\beta)$ such that

$$
\left|k^{(\beta)}(z)\right| \leq C(\alpha)|z|^{-(1+\alpha+\beta)_{+}}, \quad \forall z \neq 0 .
$$

In addition, if $\sigma=0$, we assume that $0<\alpha<2$.

(A4) Boundedness from below of $k(z)$ : there is $C_{-}>0$ such that

\section{Remark 6.1.}

$$
\forall 0<|z|<1: \quad \frac{1}{2}(k(-z)+k(z)) \geq \frac{C_{-}}{|z|^{1+\alpha}} .
$$

(i) Since $E_{\mathbb{Q}}\left[S_{t}\right]<\infty$ must hold, we have that $\int_{|z|>1} e^{z} k(z) d z<\infty$. This holds for all $k$ satisfying (A1)-(A2), due to $\eta_{+}>1$ which, from now on, is assumed to be fulfilled. 
(ii) Assumption (A2) implies that $X_{t}$ has finite moments of all orders.

(iii) Assumption (A3) is required in the analysis of the wavelet compression of the moment matrix of $k(z)$; however, it only needs to be satisfied for a finite range of $\beta$.

(iv) A Lévy process $X_{t}$ with $\sigma=0$ is called pure jump process. If $X_{t}$ is a pure jump process, we assume that it is of infinite activity, i.e.

$$
k(z) \text { satisfies (A1)-(A4) with } 0<\alpha<2 \quad(\text { if } \sigma=0) .
$$

(v) If the Lévy process is of finite activity, we assume $\sigma>0$ and that $k(z)$ satisfies (A1)-(A3) with $\alpha<0$.

6.1.1. Examples of Lévy Processes. Different parameterizations of the Lévy measure have been proposed in the literature. We mention here the Variance Gamma [31], or shortly VG process, with

$$
k(y)=C \begin{cases}|y|^{-1} e^{-\eta_{-}|y|} & \text { if } y<0 \\ |y|^{-1} e^{-\eta_{+}|y|} & \text { if } y>0\end{cases}
$$

the so-called extended Koponen family [6, 8, 11, 12, 27] (also referred to as KoBoL [6], tempered or truncated tempered stable processes or 'truncated Lévy flights' in physics, and later presented in [8] under the name of CGMY-model to fit real data), with

$$
k(y)=C\left\{\begin{array}{ll}
\frac{e^{-\eta_{-}|y|}}{|y|^{1+\alpha}} & \text { if } y<0 \\
\frac{e^{-\eta_{+}|y|}}{|y|^{1+\alpha}} & \text { if } y>0,
\end{array} \quad 0<\alpha<2,\right.
$$

the normal inverse Gaussian process (NIG) [2], and hyperbolic and generalized hyperbolic processes $[17,18,19]$. These are all examples of Lévy processes of infinite activity.

The classical Merton Model [34] is based on a drifted Brownian Motion with finitely many jumps, i.e., $X_{t}=\sigma W_{t}+\sum_{i=1}^{N_{t}} Y_{i}$. Here, $\left\{Y_{i}\right\}$ are independent, identically distributed random variables with distribution function $f(z)$, and $\left\{N_{t}\right\}$ is a Poisson process with intensity $\lambda$. The Lévy measure is given by $\nu(d z)=k(z) d z$ with $k(z)=\lambda f(z)$. With $f_{M}(z)=\left(\sqrt{2 \pi} \sigma_{M}\right)^{-1} \exp \left(-\left(z-\mu_{M}\right)^{2} /\left(2 \sigma_{M}^{2}\right)\right)$, i.e, $f_{M}$ is the normal distribution with mean $\mu_{M}$ and standard deviation $\sigma_{M}$, Merton's model is a finite intensity Lévy process which satisfies (A1)-(A3) with $\alpha=-\infty$.

In addition, we refer to the model proposed in [15] and later in [28], which assumes that

$$
f_{\text {Kou }}(z)=p_{+} M \exp (-M z) \chi_{\mathbb{R}_{+}}(z)+p_{-} G \exp (G z) \chi_{\mathbb{R}_{-}}(z),
$$

with $p_{+}+p_{-}=1$. Here, $X_{t}$ is a finite activity Lévy process with $k$ satisfying (A1)-(A3) for $\alpha=-1$.

6.1.2. Localization. The numerical solution of (6.5)-(6.6) requires the truncation of $\mathbb{R}$ to a bounded computational domain $\Omega_{R}=(-R, R)$ (note that barrier-like contracts directly lead to the PIDE on the finite interval). In the classical Black-Scholes model [5], i.e., in the absence of $\mathcal{L}^{\text {jump }}$, the localization can be effected by a simple restriction to $\Omega_{R}$ and the localization error may be estimated by local considerations. However, in the presence of the non-local operator $\mathcal{L}^{\text {jump }}$ corresponding to the jump part of the price process $\left(X_{t}\right)_{t}$, such local arguments do not apply, since the computation of the solution requires information on the pay-off on the whole real line $\mathbb{R}$.

As the transformation $u(t, \cdot) \rightarrow e^{-r t} u(t, \cdot+r t)$ reduces (6.5)-(6.6) to the case where $r=0$, we henceforth assume, without loss of generality, that $r=0$. 
Based on the observation that the excess to pay-off value $U=u-g$ decays exponentially as $|x| \rightarrow \infty$, we restrict the PIDE for the excess to pay-off value $U$ to $\Omega_{R}$. Specifically, the excess to pay-off function $U$ solves

$$
\begin{aligned}
\frac{\partial U}{\partial t}(t, x)+\mathcal{L}^{X}[U](t, x) & =-\mathcal{L}^{X}[g] & & \text { in } J \times \mathbb{R} \\
U(0, x) & =0 & & \text { in } \mathbb{R} .
\end{aligned}
$$

Now, rather then solving (6.15)-(6.16), we solve the truncated problem in $(0, T) \times \Omega_{R}$

$$
\begin{aligned}
& \frac{\partial U_{R}}{\partial t}+\mathcal{L}_{R}\left[U_{R}\right]=-\left.\mathcal{L}^{X}[g]\right|_{\Omega_{R}} \quad \text { in } \quad J \times \Omega_{R} \\
& U_{R}(t, x)=0 \quad \text { in } \quad J \times \mathbb{R} \backslash \Omega_{R} \\
& U_{R}(0)=0 \quad \text { in } \Omega_{R},
\end{aligned}
$$

with $\mathcal{L}_{R}$ being the restriction of $\mathcal{L}^{X}$ to $\Omega_{R}$. Note that, in contrast to the standard BlackScholes case $\left(\mathcal{L}^{\text {jump }}=0\right)$, the non-local operator $\mathcal{L}^{\text {jump }}$ requires the pay-off function $g$ to be specified also outside the domain $\Omega_{R}$.

By [33, Proposition 3.6], the right-hand side $-\mathcal{L}^{X}[g]$ is given by

$$
-\mathcal{L}^{X}[g]=\sigma^{2} / 2 K \delta_{\log (K)}-\mathcal{L}^{\text {jump }}[g],
$$

with $\mathcal{L}^{\text {jump }}[g] \in C^{\infty}(\mathbb{R} \backslash \log (K)) \cap L_{\text {loc }}^{1}(\mathbb{R})$ decaying exponentially at infinity:

$$
-\mathcal{L}^{\text {jump }}[g] \leq C e^{-\eta_{\mp}|x|} \text { as } \pm x \rightarrow \infty .
$$

In addition, the excess to pay-off function $U$ decays exponentially at infinity, and by [33, Theorem 4.1], the error $U_{R}-U$ caused by the localization is exponentially small as $R \rightarrow$ $\infty$.

6.1.3. Parabolic Setting. The localized problem (6.17)-(6.19) can be cast in the variational setting (2.1)-(2.2) of Section 2. To show this, let $\mathcal{A}:=\mathcal{L}_{R}$ and $a(\cdot, \cdot)$ denote the bilinear form induced by $\mathcal{A}$,

$$
a(\varphi, \psi):=\int_{\Omega_{R}} \mathcal{A}[\varphi](x) \psi(x) d x, \quad \forall \varphi, \psi \in C_{0}^{\infty}\left(\Omega_{R}\right) .
$$

If $\sigma>0$, the price process contains a diffusion component and the order of the operator $\mathcal{L}^{X}$ is 2 . Then $a(\cdot, \cdot)$ can be extended continuously to $V \times V$ with $V=H_{0}^{1}\left(\Omega_{R}\right)$ and the following Gårding inequality holds: there exist $c_{1}, c_{2}>0$ such that

$$
a(v, v) \geq c_{1}\|v\|_{H^{1}\left(\Omega_{R}\right)}^{2}-c_{2}\|v\|_{L^{2}\left(\Omega_{R}\right)}^{2}, \quad \forall v \in V .
$$

In the pure jump case, $\sigma=0$, the order of $\mathcal{L}^{X}$ is in general $\max \{1, \alpha\}$ due to the presence of the drift term in $\mathcal{L}^{X}$. There holds:

Proposition 6.2. Assume that $X_{t}$ is a pure jump Lévy process (i.e. $\sigma=0$ ) with Lévy density $k$ satisfying (A1)-(A4) for some $0<\alpha<2$. Then, there exist two positive constants $c_{1}=c_{1}(R)>0$ and $c_{2}=c_{2}(R)>0$ such that

$$
\forall u \in \tilde{H}^{\alpha / 2}\left(\Omega_{R}\right): a(u, u) \geq c_{1}\|u\|_{\tilde{H}^{\alpha / 2}\left(\Omega_{R}\right)}^{2}-c_{2}\|u\|_{L^{2}\left(\Omega_{R}\right)}^{2},
$$

i.e., on $\tilde{H}^{\alpha / 2}\left(\Omega_{R}\right)$, the bilinear form a $(\cdot, \cdot)$ satisfies a Gårding inequality as in (2.2).

Proof. See [32]. 
Remark 6.3. Proposition 6.2 states a Gårding inequality (2.2) in the pure jump case $\sigma=0$ for all jump intensities $\alpha \in(0,2)$. However, if the term $\left(u^{\prime}, v\right)$ is present, the continuity (2.1) on $V=\tilde{H}^{\alpha / 2}$ holds only if $1 \leq \alpha \leq 2$, see [33].

In order to prove (2.1) in the case $\sigma=0$ and $0<\alpha<1$, it is important to remove the 'drift' term so that the transformed equation satisfies the Gårding inequality (2.2) in $\tilde{H}^{\alpha / 2}\left(\Omega_{R}\right)$ as well as the continuity estimate (2.1). Let $\mathcal{A}^{\text {jump }}:=\left.\mathcal{L}^{\text {jump }}\right|_{\Omega_{R}}$ be the restriction of the integro-differential operator $\mathcal{L}^{\text {jump }}$ to $\Omega_{R}$. By reduction to the 'transformed' variables, we assume again that $r=0$, together with $0<\alpha<1$. Then,

$$
\mathcal{A}[u]=-\frac{\sigma^{2}}{2} u^{\prime \prime}+\frac{\sigma^{2}}{2} u^{\prime}+\mathcal{A}^{\text {jump }}[u] .
$$

Next, the operator $\mathcal{A}^{\text {jump }}$ can be written as a sum of a first order term and an integrodifferential operator $\mathcal{C}$ which is continuous and coercive on $\tilde{H}^{\alpha / 2}\left(\Omega_{R}\right)$ :

$$
\left\langle\mathcal{A}^{\text {jump }} \varphi, \psi\right\rangle=c_{1}\left\langle\varphi^{\prime}, \psi\right\rangle+\langle\mathcal{C}[\varphi], \psi\rangle, \quad \forall \varphi, \psi \in C_{0}^{\infty}\left(\Omega_{R}\right),
$$

with

$$
c_{1}:=\int_{\mathbb{R}}\left(e^{y}-1\right) k(y) d y .
$$

The operator $\mathcal{C}$ is defined by

$$
\langle\mathcal{C} u, v\rangle:=-\int_{\mathbb{R}} \int_{\mathbb{R}} k(y-x)(\tilde{u}(y)-\tilde{u}(x)) \tilde{v}(x) d y d x, \quad \forall u, v \in \tilde{H}^{\alpha / 2}\left(\Omega_{R}\right),
$$

where, by $\tilde{v}$, we denote the extension by zero of $v$ to $\mathbb{R}$. Then, there exist constants $C, C_{1}, C_{2}>0$ such that

$$
\begin{array}{lll}
\langle\mathcal{C} u, v\rangle \leq C\|u\|_{\tilde{H}^{\alpha / 2}\left(\Omega_{R}\right)}\|v\|_{\tilde{H}^{\alpha / 2}\left(\Omega_{R}\right)}, & & \forall u, v \in \tilde{H}^{\alpha / 2}\left(\Omega_{R}\right), \\
\langle\mathcal{C} u, u\rangle \geq C_{1}\|u\|_{\tilde{H}^{\alpha / 2}\left(\Omega_{R}\right)}^{2}-C_{2}\|u\|_{L^{2}\left(\Omega_{R}\right)}^{2}, & & \forall u \in \tilde{H}^{\alpha / 2}\left(\Omega_{R}\right) .
\end{array}
$$

We have seen that the presence of the drift term $c_{1} \varphi^{\prime}$ obstructs the continuity of the form $\left\langle\mathcal{A}^{\text {jump }}[\varphi], \psi\right\rangle$. We remove this term by the transformation

$$
U(t, x)=V\left(t, x-\left(\sigma^{2} / 2+c_{1}\right) t\right)
$$

which yields the parabolic problem

$$
\begin{aligned}
\frac{\partial V}{\partial t}(t, x)-\frac{\sigma^{2}}{2} \frac{\partial^{2} V}{\partial x^{2}}+\mathcal{C}[V](t, x) & =\left(-\left.\mathcal{L}^{X}[g]\right|_{\Omega_{R}}\right)\left(x+\left(\sigma^{2} / 2+c_{1}\right) t\right) \\
V(0, x) & =0 .
\end{aligned}
$$

Remark 6.4. We note that the numerical scheme proposed in this paper exploits the parabolic nature of (6.5) and, furthermore, that a dominant first order term may be a source of instabilities even in the case $\sigma \neq 0$. Therefore, the removal of the 'drift' is also of interest from the point of view of numerical approximation.

Due to our previous considerations, $a(\cdot, \cdot): V \times V \rightarrow \mathbb{R}$ satisfies (2.1)-(2.2) (cf. Proposition 6.2) in the triple $V \stackrel{d}{\hookrightarrow} L^{2}\left(\Omega_{R}\right) \stackrel{d}{\hookrightarrow} V^{*}$ with

$$
V=\tilde{H}^{\rho / 2}\left(\Omega_{R}\right)
$$

where the order $\rho$ is given by

$$
\rho= \begin{cases}2 & \text { if } \sigma>0 \\ \alpha & \text { if } \sigma=0\end{cases}
$$


For any $v \in H^{\rho / 2}$, let $P_{a}^{V} v$ denote the $V$-projection of $v$ with respect to $a(\cdot, \cdot)$, i.e. $a\left(v-P_{a}^{V} v, w\right)=0$, for all $w \in V$. By the continuity of $a(\cdot, \cdot)$, it holds that

$$
\left\|P_{a}^{V} v\right\|_{V} \leq\|v\|_{H^{\rho / 2}\left(\Omega_{R}\right)}, \quad \forall v \in H^{\rho / 2}\left(\Omega_{R}\right) .
$$

The variational formulation of (6.17)-(6.19) reads: find $U_{R} \in L^{2}(J, V) \cap H^{1}\left(J, V^{*}\right)$ such that

$$
\begin{aligned}
\left(\frac{\partial U_{R}}{\partial t}(t, \cdot), v\right)_{L^{2}\left(\Omega_{R}\right)}+a\left(U_{R}(t, \cdot), v\right) & =-\left\langle\mathcal{L}^{X}[g], v\right\rangle_{V^{*} \times V}, \quad \forall v \in V, \\
U_{R}(0, \cdot) & =0 .
\end{aligned}
$$

Obviously, (6.26)-(6.27) is equivalent to solving

$$
\begin{aligned}
\left(\frac{\partial u_{R}}{\partial t}(t, \cdot), v\right)_{L^{2}\left(\Omega_{R}\right)}+a\left(u_{R}(t, \cdot), v\right) & =0 \quad \forall v \in V \\
u_{R}(0, \cdot) & =u_{0}:=P_{a}^{V} g,
\end{aligned}
$$

where, evidently, $u_{R}=P_{a}^{V} g+U_{R}$.

Hence, dropping the subscript $R$ to simplify notations and referring to (6.28)-(6.29), we are now in the setting of Section 2 with $u_{0} \in V$.

6.2. Non-Translation-Invariant Operators. The numerical method presented in this paper (see Sections 3-6) applies also to the more general case where the log-price process $X_{t}$ is a Feller process [33] with increments $X_{t}-X_{s}$ that are no longer independent of $X_{s}$ for $t>s$. Instead of the translation-invariant operator $\mathcal{L}^{X}$ from (6.7)-(6.8), we now consider a more general operator $\mathcal{L}^{X}$

$$
\mathcal{L}^{X}[\varphi](x):=-\frac{\sigma(x)^{2}}{2} \varphi^{\prime \prime}(x)+\left(\frac{\sigma(x)^{2}}{2}-r\right) \varphi^{\prime}(x)+r \varphi+\mathcal{L}^{\mathrm{jump}}[\varphi](x),
$$

where the integral operator $\mathcal{L}^{\text {jump }}$ is given by

$$
\mathcal{L}^{\text {jump }}[\phi](x):=-\int_{\mathbb{R}}\left(\phi(y)-\phi(x)-\left(e^{y-x}-1\right) \phi^{\prime}(x)\right) k(x, y-x) d y .
$$

We assume

$$
0<\sigma_{0} \leq \sigma(x) \leq \sigma_{1} \quad \forall x \in \mathbb{R}
$$

and that $k(x, z)$ satisfies the assumptions (A1)-(A2) uniformly with respect to $x \in \mathbb{R}$. Instead of (A3), we require the Calderón-Zygmund estimates (2.6). Furthermore, in the case $\sigma=0$, we assume (A4) uniformly with respect to $x \in \Omega_{R}$.

If $\sigma>0$ or $\alpha \geq 1$ the corresponding bilinear form $a$ satisfies (2.1)-(2.2). If $\sigma=0$ and $\alpha<1$, we need to assume that we can transform the problem such that $a$ satisfies (2.1).

As an example, we refer to [3] for a pure jump Feller process of normal inverse Gaussian type satisfying (A1)-(A4) with $\alpha=1$ uniformly with respect to $x$.

\section{CONCluding Remarks}

In the present paper, we have considered the efficient numerical solution of parabolic integro-differential equations where the spatial operator is a classical pseudo-differential operator of order $\max \{1, \alpha\}<2$. Such operators arise as infinitesimal generators of semigroups generated by certain stochastic processes with a quadratic, pure jump component. Using the variable order timestepping and geometric step size reduction towards $t=0$, analyticity of the process' semigroup and wavelet compression of the infinitesimal generator, we have shown how to compute approximate expectations over all paths with accuracy 
$\mathcal{O}\left(N^{-p-1}\right)$ for any degree $p \geq 1$ of the wavelets and essentially, i.e. up to logarithmic terms, $\mathcal{O}(N)$ memory and $\mathcal{O}(N)$ float point operations, thereby matching the complexity of FFT-based methods [9]. Unlike FFT-based methods, however, the methods developed here apply to nonstationary processes as well as to additive process with analytic time dependence in the characteristic triple. Applications to option pricing in finance have been discussed.

\section{REFERENCES}

[1] R. A. Adams. Sobolev Spaces. Academic Press, 1978. New York.

[2] O. Barndorff-Nielsen. Processes of normal inverse gaussian type. Finance and Stochastics, 2:41-68, 1998.

[3] O. Barndorff-Nielsen and S. Levendorski. Feller processes of normal inverse gaussian type. Quantitative Finance, 1:318-331, 2001.

[4] J. Bertoin. Lévy processes. Cambridge Univ. Press, 1996.

[5] F. Black and M. Scholes. The pricing of options and corporate liabilities. Journal of Political Economy, 81:637-654, 1973.

[6] S. Boyarchenko and S. Levendorski. Option pricing for truncated Lévy processes. Int. Journ. Theor. and Appl. Finance, 3(3):549-552, 2000.

[7] S.I. Boyarchenko and S.Z. Levendorskii. Non-Gaussian Merton-Black-Scholes Theory. Advanced Series on Statistical Science \& Applied Probability 9, World Scientifi c, 2002.

[8] P. Carr, H. Geman, and D. B. Madan. The fi ne structure of asset returns: An empirical investigation. Journal of Business, 2002.

[9] P. Carr and D. Madan. Option valuation using the fft. J. Comp. Finance, 2:61-73, 1999.

[10] A. Cohen. Wavelet methods for operator equations. In Handbook of Numerical Analysis. Elsevier, 2001. To appear.

[11] R. Cont, J. P. Bouchaud, and M. Potters. Scaling in fi nancial data: stable laws and beyond. In B. Dubrulle, F. Graner, and D. Sornette, editors, Scale invariance and beyond. Springer Berlin, 1997.

[12] R. Cont and P. Tankov. Financial modelling with jump processes. Chapman and Hall/CRC Press, 2004.

[13] R. Cont and E. Voltchkova. A fi nite-difference scheme for option pricing in jump-diffusion and exponential lévy models. Rapport Interne CMAP, 513, 2003.

[14] W. Dahmen, A. Kunoth, and K. Urban. Biorthogonal spline wavelets on the interval—stability and moment conditions. Appl. Comput. Harmon. Anal., 6(2):132-196, 1999.

[15] D. Duffi e, J. Pand, and K. Singleton. Transform analysis and option pricing for affi ne jump-diffusions. Econometrica, 68:1343-1376, 2000.

[16] E. B. Dynkin. Theory of Markov processes. Pergamon Press, Oxford, 1960.

[17] E. Eberlein. Application of generalized hyperbolic Lévy motions to fi nance. In O. E. Barndorff-Nielsen, T. Mikosch, and S. Resnick, editors, Lévy Processes: Theory and Applications, pages 319-337. Birkhäuser, 2001.

[18] E. Eberlein and U. Keller. Hyperbolic distributions in fi nance. Bernoulli, 1:281-299, 1995.

[19] E. Eberlein, U. Keller, and K. Prause. New insights into smile, mispricing and value at risk: the hyperbolic model. Journal of Business, 71(3):371-405, 1998.

[20] R. P. Feynman and A. Hibbs. Quantum Mechanics and Path Integrals. McGraw Hill, New York, 1965.

[21] M. Freidlin. Functional integration and partial differential equations. Annals of Mathematics Studies. Princeton University Press, 1985.

[22] H. Fujita and T. Suzuki. Handbook of Numerical Analysis II: Finite Element Methods I, chapter 'Evolution Problems', pages 789-928. North-Holland, 1991.

[23] N. Jacob. Pseudo-Differential Operators and Markov Processes. Vol. 1: Fourier Analysis and Semigroups. Imperial College Press, London, 2001.

[24] N. Jacob. Pseudo-Differential Operators and Markov Processes. Vol. 2: Generators and their Potential Theory. Imperial College Press, London, 2002.

[25] M. Kac. On some connections between probability theory and differential and integral equations. In $2 n d$ Berkeley Symp. on Math. Stat. and Prob. Univ. of California Press: Berkeley and Los Angeles, 1951.

[26] I. Karatzas and S. E. Shreve. Methods of Mathematical Finance. Springer, 1999.

[27] I. Koponen. Analytic approach to the problem of convergence of truncated lévy fights towards the gaussian stochastic process. Phys. Rev. E, 52:1197-1199, 1995.

[28] G. Kou. A jump diffusion model for option pricing. Mangement Science, 48:1086-1101, 2002. 
[29] D. Lamberton and B. Lapeyre. Introduction to Stochastic Calculus Applied to Finance. Chapman \& Hall, 1997.

[30] J. L. Lions and E. Magenes. Non-homogeneous boundary value problems and applications. Springer Berlin, 1972.

[31] D. B. Madan, P. Carr, and E. Chang. The variance gamma process and option pricing. European Finance Review, 2(79-105), 1998.

[32] A.-M. Matache, C. Schwab, and T. P. Wihler. Fast numerical solution of parabolic integro-differential equations with applications in fi nance. Technical Report \# 1954, Institute for Mathematics and its Applications (IMA), University of Minnesota, January 2004.

[33] A.-M. Matache, T. von Petersdorff, and C. Schwab. Fast deterministic pricing of options on Lévy driven assets. Mathematical Modelling and Numerical Analysis, 38(1):37-71, 2004.

[34] R.C. Merton. Option pricing when the underlying stocks are discontinuous. J. Financ. Econ., 5:125-144, 1976.

[35] D. Nualart and W. Schoutens. Backward stochastic differential equations and Feynman-Kac formula for Lévy processes, with applications in fi nance. Bernoulli, 7(5):761-776, 2001.

[36] K.-I. Sato. Lévy Processes and Infinitely Divisible Distributions. Cambridge University Press, 1999.

[37] R. Schneider. Multiskalen- und Wavelet-Matrixkompression. Analysisbasierte Methoden zur effizienten Lösung grosser vollbesetzter Gleichungssysteme. Advances in Numerical Mathematics. B. G. Teubner, Stuttgart, 1998.

[38] D. Schötzau and C. Schwab. Time discretization of parabolic problems by the $h p$-version of the discontinuous Galerkin fi nite element method. SIAM J. Num. Anal., 38:837-875, 2000.

[39] D. Schötzau and C. Schwab. $h p$-discontinuous Galerkin time-stepping for parabolic problems. C.R.Acad. Sci. Paris, Série I:1121-1126, 2001.

[40] H. Triebel. Interpolation Theory, Function Spaces, Differential Operators. J.A. Barth Publishers, Heidelberg-Leipzig, 2nd edition, 1995.

[41] T. von Petersdorff and C. Schwab. Fully discrete multiscale Galerkin BEM. In Multiresolution Analysis and Partial Differential Equations, W. Dahmen, P. Kurdila and P. Oswald (Eds.), volume 6 of Wavelet Analysis and Its Applications, pages 287-346. Academic Press, New York, 1997.

[42] T. von Petersdorff and C. Schwab. Wavelet discretizations of parabolic integro-differential equations. SIAM J. Numer. Anal., 41(1):159-180, 2003.

[43] T. von Petersdorff and C. Schwab. Numerical solution of parabolic equations in high dimensions. Mathematical Modelling and Numerical Analysis, 38(1):93-128, 2004.

SEMinAR FOr ApPliEd MATHEMATICS AND RISKLAB, ETH ZÜRICH, 8092 ZÜRICH, SWITZERLAND. E-mail address: amatache@math.ethz.ch

SEMINAR FOR APPliEd MATHEMATICS, ETH ZÜRICH, 8092 ZÜRICH, SWITZERLAND.

E-mail address: schwab@math. ethz.ch

School of Mathematics, University of Minnesota, Minneapolis MN, 55455, USA.

E-mail address: wihleremath.umn.edu 